Interfaces and Free Boundaries 15 (2013), 239-263

DOI $10.4171 / \mathrm{IFB} / 302$

\title{
Two-phase flow in rotating Hele-Shaw cells with Coriolis effects
}

\author{
JOACHIM ESCHER \\ Leibniz Universität Hannover, Institut für Angewandte Mathematik, \\ Welfengarten 1, 30167 Hannover, Germany \\ E-mail: escher@ifam.uni-hannover.de \\ PATRICK GUIDOTTI \\ University of California, Irvine, Department of Mathematics, \\ 340 Rowland Hall, Irvine, CA 92697-3875, USA \\ E-mail: gpatrick@math.uci.edu \\ CHRISTOPH WALKER \\ Leibniz Universität Hannover, Institut für Angewandte Mathematik, \\ Welfengarten 1, 30167 Hannover, Germany \\ E-mail:walker@ifam.uni-hannover.de
}

[Received 9 October 2012 and in revised form 14 May 2013]

\begin{abstract}
The free boundary problem of a two phase flow in a rotating Hele-Shaw cell with Coriolis effects is studied. Existence and uniqueness of solutions near spheres is established, and the asymptotic stability and instability of the trivial solution is characterized in dependence on the fluid densities.
\end{abstract}

2010 Mathematics Subject Classification: Primary 35K90, 35Q35, 42A16.

Keywords: Two phase flow, free boundary problem, well-posedness, stability of equilibria.

\section{Introduction}

The motion of one or two fluids confined to a narrow gap between two parallel plates is an interesting problem with a long history. It is the classical set up of the so-called Hele-Shaw problem [12]. It is well-known that instability driven pattern formation such as fingering can occur under appropriate assumptions on the viscosity of the fluids [14] and on the surface tension at their interface. There is a vast empirical and theoretical literature concerning this classical problem. From the purely mathematical point of view the problem has been extensively studied in its original formulation as a one [5, 6, 8, 9] and as a two-phase [18] problem and, more recently, also for one fluid in the case of rotating plates [7]. The focus is here on the two-phase problem with rotating plates and including the effects of Coriolis forces. This setting has recently been considered in the physical literature by a variety of authors [1, 10, 17]. It first appeared in [15] where the effects of rotation were introduced in a ad-hoc fashion into Darcy's law for the one-phase problem

$$
\nabla p=-\frac{12 \eta}{b^{2}} \vec{v}+\varrho \omega^{2} \vec{x}+2 \varrho \omega \vec{z} \times \vec{v},
$$

where $\vec{z}$ is the axis of rotation, $b$ is the plate spacing, $\vec{x}$ is the two-dimensional position vector, $\omega$ is the (constant) angular velocity of the plates, whereas $\vec{v}, p, \eta$, and $\varrho$ are the fluid's velocity, pressure, viscosity, and density, respectively. The last term accounts for Coriolis' force. While most of the 
literature hitherto neglected this force, recent studies performed a model derivation for a one-phase Hele-Shaw type model with Coriolis force by means of a standard gap averaging technique starting from Navier-Stokes' equations, cf. [16, 17]. The authors of [17] observe that the effects due to the Coriolis term in their equations can be larger than the inertial terms typically neglected in small Reynolds number type reductions. They also point out the fact that simpler ad-hoc models used earlier, while qualitatively similar, do eventually lead to a different and inaccurate prediction of the growth rate of the unstable modes involved in the fingering phenomenon.

More recently, the effects of a Coriolis term on the fingering patterns in the rotating two-phase Hele-Shaw problem have been studied in $[1,10]$ where the formal linear stability analyses of $[15$, 17] for the one-phase problem are extended to the weakly nonlinear case by the use of formal expansions and to the fully nonlinear regime by means of numerical simulations. Previous numerical and experimental results included $[4,16]$, whereas the practical relevance of the problem is attested by a number of publications cited in [1] for that very purpose.

In this paper local existence of a unique classical solution for nearly circular initial interfaces is established for the general rotating two-phase Hele-Shaw problem with Coriolis effects in the formulation proposed in [1, 10], which is, in turn, based on a derivation similar to that of [17] for the single phase case. It leads to the following generalized Darcy's law

$$
\nabla P_{j}=-\alpha_{j} \vec{v}_{j}+\beta_{j} \vec{z} \times \vec{v}_{j},
$$

for the velocities of the inner and outer fluids ( $j=\mathrm{i}, \mathrm{o}$ ), for constants $\alpha_{j}$ and $\beta_{j}$ defined below and for the pressure $P_{j}$ related to the hydrostatic pressure $p_{j}$ via

$$
P_{j}=p_{j}-\frac{\varrho_{j} \omega^{2}}{2}|x|^{2} .
$$

The use of the unusual pressure $P_{j}$ yields some useful simplifications as will soon become apparent. Rigorous stability results are also obtained on near circularity assumptions for the initial data by resorting to a general principle of linearized stability. While the circular steady state is exponentially asymptotically stable ${ }^{2}$ when the outer fluid is denser, it becomes unstable if this relation is reversed. We shall also see that the equilibrium problems for two phases and one phase are essentially equal. The main techniques used are a transformation of the original free boundary problem to a fixed domain one, a decoupling of the system and a reduction to a single nonlinear and nonlocal evolution for the interface separating the two fluids, and parabolic optimal regularity results in little Hölder spaces.

Related previous mathematical results include the existence, stability, and bifurcation analysis of [7] in the rotating one-phase problem and the global existence and stability of smooth solutions obtained by [18] for nearly circular initial interfaces in the non-rotating two-phase case.

\section{Governing equations and main results}

We first give a short justification for the mathematical formulation of the physical problem of a rotating Hele-Shaw cell including Coriolis effects. Further details regarding the modeling can be found in $[1,10]$. We then end this section with the main results on existence of solutions to the corresponding governing equations and stability properties of the trivial solution.

\footnotetext{
${ }^{2}$ Our analytical approach reveals a precise description of the exponential decay in terms of the physical parameters, in particular of the Coriolis terms, see Section 4.
} 


\subsection{Governing equations}

Consider a circular Hele-Shaw cell of radius $R \geqslant 2$ and very small gap width $b>0$, rotating clockwise around the $z$-axis with a constant angular velocity $\omega>0$. The cell is assumed to contain two immiscible, incompressible, viscous fluids with densities $\varrho_{j}>0$ and viscosities $\eta_{j}>0$, where $j=i$ labels the inner and $j=o$ the outer fluid, respectively. The surface tension between the two fluids is given by $\sigma>0$. The rotating coordinate system is defined in such a way that its origin is located at the cell center and that rotation is perpendicular to the vector $\vec{z}=(0,0,1)$. Let $\Omega^{j}(t)$ denote the region of space occupied by fluid $j \in\{\mathrm{i}, \mathrm{o}\}$ at time $t$, and let $\Gamma(t)$ denote the sharp interface separating the two fluids. The unit normal vector $v_{\Gamma(t)}$ to $\Gamma(t)$ is assumed to point from $\Omega^{\mathrm{i}}(t)$ to $\Omega^{\mathrm{o}}(t)$.

The basic hydrodynamic equation of the system is a generalized Darcy's law relating the pressure fields $P_{j}=P_{j}(t)$ to the two-dimensional gap-averaged flow velocities $\vec{v}_{j}=\vec{v}_{j}(t)$ through

$$
\nabla P_{j}=-\alpha_{j} \vec{v}_{j}+\beta_{j}\left(\vec{z} \times \vec{v}_{j}\right) \text { in } \Omega^{j}(t) .
$$

The numbers

$$
\alpha_{j}:=\frac{12 \eta_{j}}{b^{2}} E_{j}, \quad \beta_{j}:=\frac{12 \eta_{j}}{b^{2}} F_{j}
$$

depend linearly on the Coriolis force terms $E_{j}>0$ and $F_{j} \geqslant 0$, respectively. The latter, in turn, depend on the rotational Reynolds number $R e_{j}=\varrho_{j} \omega b^{2} / 12 \eta_{j}$ (see [1]). If Coriolis effects are neglected, then $E_{j}=1$ and $F_{j}=0$, so (2.1) reduces to the usual Darcy's law. Throughout this paper we shall assume that $\alpha_{j}>0$ and $\beta_{j} \geqslant 0$. Incompressibility of the fluids is expressed by

$$
\operatorname{div} \vec{v}_{j}=0 \text { in } \Omega^{j}(t),
$$

while the interface dynamics is governed by the normal stress balance

$$
P_{\mathrm{i}}-P_{\mathrm{o}}=\sigma \kappa_{\Gamma(t)}+\left(\gamma_{\mathrm{o}}-\gamma_{\mathrm{i}}\right)|x|^{2} \quad \text { on } \quad \Gamma(t),
$$

and the kinematic boundary condition

$$
V=\vec{v}_{\mathrm{i}} \cdot v_{\Gamma}=\vec{v}_{\mathrm{o}} \cdot v_{\Gamma} \quad \text { on } \quad \Gamma(t) .
$$

Here, $\gamma_{j}:=\varrho_{j} \omega^{2} / 2$ whereas $\kappa_{\Gamma(t)}$ denotes the curvature of $\Gamma(t)$ which is taken to be positive if $\Omega^{\mathrm{i}}(t)$ is convex. The normal velocity of the interface is given by $V=V(t)$. On the outer boundary of the cell a no-slip condition

$$
\vec{v}_{\mathrm{o}} \cdot x=0 \quad \text { on } \quad[|x|=R]
$$

is imposed.

Observe that the inner and outer fluids' masses are conserved since, by Reynold's transport theorem, one has that

$$
\frac{\mathrm{d}}{\mathrm{d} t} \int_{\Omega^{\circ}(t)} \mathrm{d} x=-\int_{\Gamma(t)} \vec{v}_{\mathrm{o}} \cdot v_{\Gamma} \mathrm{d} \sigma+\int_{[|x|=R]} \vec{v}_{\mathrm{o}} \cdot \frac{x}{R} \mathrm{~d} \sigma
$$

and

$$
\frac{\mathrm{d}}{\mathrm{d} t} \int_{\Omega^{\mathrm{i}}(t)} \mathrm{d} x=\int_{\Gamma(t)} \vec{v}_{\mathrm{i}} \cdot v_{\Gamma} \mathrm{d} \sigma
$$

which are both zero in view of Gauss' Theorem and (2.2). Since $\operatorname{rot}\left(\vec{z} \times \vec{v}_{j}\right)=\left(0,0, \operatorname{div} \vec{v}_{j}\right)$ we also note that the flows are irrotational in the bulk, i.e., $\operatorname{rot} \vec{v}_{j}=0$ in $\Omega^{j}(t)$, due to (2.1) and (2.2). 


\subsection{The system in terms of $P_{j}$}

The governing equations in terms of $P_{j}$ are obtained by taking the divergence on both sides of (2.1) which yields

$$
\Delta P_{j}=0 \text { in } \Omega^{j}(t),
$$

making use of (2.2) and observing that $\operatorname{rot} \vec{v}_{j}=0$ implies $\operatorname{div}\left(\vec{z} \times \vec{v}_{j}\right)=0$. As for the kinematic boundary condition (2.4), we solve (2.1) for $\vec{v}_{j}$ to get

$$
\vec{v}_{j}=\frac{1}{\left|\Theta_{j}\right|^{2}}\left(-\alpha_{j} \nabla P_{j}-\beta_{j}\left(\vec{z} \times \nabla P_{j}\right)\right)
$$

with complex numbers $\Theta_{j}:=\alpha_{j}+i \beta_{j}$. Then, defining the tangent vector $\tau_{\Gamma}:=-\left(\vec{z} \times v_{\Gamma}\right)$ to $\Gamma$, we obtain

$$
\vec{v}_{j} \cdot v_{\Gamma}=\frac{1}{\left|\Theta_{j}\right|^{2}}\left(-\alpha_{j} \partial_{\nu_{\Gamma}} P_{j}-\beta_{j} \partial_{\tau_{\Gamma}} P_{j}\right)
$$

Whence (2.4) and (2.5) become

$$
-V=\frac{1}{\left|\Theta_{\mathrm{i}}\right|^{2}}\left(\alpha_{\mathrm{i}} \partial_{\nu_{\Gamma}} P_{\mathrm{i}}+\beta_{\mathrm{i}} \partial_{\tau_{\Gamma}} P_{\mathrm{i}}\right)=\frac{1}{\left|\Theta_{\mathrm{o}}\right|^{2}}\left(\alpha_{\mathrm{o}} \partial_{\nu_{\Gamma}} P_{\mathrm{o}}+\beta_{\mathrm{o}} \partial_{\tau_{\Gamma}} P_{\mathrm{o}}\right) \quad \text { on } \quad \Gamma(t),
$$

and

$$
\alpha_{\mathrm{o}} \partial_{v} P_{\mathrm{o}}+\beta_{\mathrm{o}} \partial_{\tau} P_{\mathrm{o}}=0 \quad \text { on } \quad[|x|=R],
$$

respectively, where $v$ denotes the unit outer normal vector and $\tau$ the corresponding tangential vector on the cell boundary $[|x|=R]$. Therefore, we arrive at the following free boundary problem for the pressures $P_{j}$

$$
\begin{aligned}
\Delta P_{j} & =0 & & \text { in } \Omega^{j}(t), j=\mathrm{i}, \mathrm{o}, \\
P_{\mathrm{i}}-P_{\mathrm{o}} & =\sigma \kappa_{\Gamma(t)}+\left(\gamma_{\mathrm{o}}-\gamma_{\mathrm{i}}\right)|x|^{2} & & \text { on } \Gamma(t), \\
-V=\frac{1}{\left|\Theta_{\mathrm{i}}\right|^{2}}\left(\alpha_{\mathrm{i}} \partial_{\nu_{\Gamma}} P_{\mathrm{i}}+\beta_{\mathrm{i}} \partial_{\tau_{\Gamma}} P_{\mathrm{i}}\right) & =\frac{1}{\left|\Theta_{\mathrm{o}}\right|^{2}}\left(\alpha_{\mathrm{o}} \partial_{\nu_{\Gamma}} P_{\mathrm{o}}+\beta_{\mathrm{o}} \partial_{\tau_{\Gamma}} P_{\mathrm{o}}\right) & & \text { on } \Gamma(t), \\
\alpha_{\mathrm{o}} \partial_{\nu} P_{\mathrm{o}}+\beta_{\mathrm{o}} \partial_{\tau} P_{\mathrm{o}} & =0 & & \text { on }[|x|=R],
\end{aligned}
$$

for $t>0$, complemented with an initial surface $\Gamma(0)=\Gamma^{0}$. Since only derivatives and the difference of the two pressures enter the system, uniqueness can only be expected up to additive constants for them. As we shall later see, this is one of the obstacles that need to be overcome from a mathematical viewpoint.

\subsection{Main theorems}

To give a precise formulation of our mathematical results on (2.6)-(2.9), we parametrize the boundary $\Gamma(t)$ over the unit sphere $\mathbb{S}^{1}:=\left\{x \in \mathbb{R}^{2} ;|x|=1\right\}$. To this end we introduce, for $s \geqslant 0$, so-called little Hölder spaces $h^{s}(U)$ over an open subset $U$ of $\mathbb{R}^{n}$ as the closure of $B U C^{\infty}(U)$ in $B U C^{s}(U)$. Here $B U C^{s}(U)$ consists of all functions $f: U \rightarrow \mathbb{R}$ with bounded and uniformly continuous derivatives up to order $[s]$ and with uniformly $(s-[s])$-Hölder continuous derivatives of order [s]. If $M$ is a (sufficiently smooth) submanifold of $\mathbb{R}^{n}$, we define $h^{s}(M)$ by means of an 
atlas for $M$ in the canonical way. In the following we shall identify a function $h: \mathbb{S}^{1} \rightarrow \mathbb{R}$ with the function $\bar{h}:[0,2 \pi] \rightarrow \mathbb{R}$ given by $\bar{h}(\theta):=h\left(e^{i \theta}\right)$ for $0 \leqslant \theta \leqslant 2 \pi$. The bar will often be dropped as no confusion seems likely.

Fix $a \in(0,1 / 4)$ and $\delta \in(0,1)$ and set

$$
v:=\left\{\rho \in h^{4+\delta}\left(\mathbb{S}^{1}\right) ;\|\rho\|_{\infty}<a\right\},
$$

and, for $\rho \in V$,

$$
\Omega_{\rho}^{\mathrm{i}}:=\left\{x \in \mathbb{R}^{2} \backslash\{0\} ;|x|<1+\rho\left(\frac{x}{|x|}\right)\right\} \cup\{0\}, \quad \Omega_{\rho}^{\mathrm{o}}:=\Omega \backslash \overline{\Omega_{\rho}^{\mathrm{i}}}
$$

with $\Omega:=\mathbb{B}(0, R)$ where, we recall, $R \geqslant 2$. Then

$$
\Gamma_{\rho}:=\left\{x \in \mathbb{R}^{2} ;|x|=1+\rho\left(\frac{x}{|x|}\right)\right\}=\left\{[1+\rho(y)] y ; y \in \mathbb{S}^{1}\right\}
$$

separates the domains $\Omega_{\rho}^{\mathrm{i}}$ and $\Omega_{\rho}^{\mathrm{o}}$. Since $\Gamma_{\rho}$ can be described as the zero level set of

$$
N_{\rho}(x):=|x|-1-\rho\left(\frac{x}{|x|}\right), \quad 3 / 4<|x|<5 / 4,
$$

with $N_{\rho}<0$ in $\Omega_{\rho}^{\mathrm{i}} \cap[3 / 4<|x|<5 / 4]$, the unit normal $v_{\rho}(x)$ at $x \in \Gamma_{\rho}$ pointing from $\Omega_{\rho}^{\mathrm{i}}$ to $\Omega_{\rho}^{\mathrm{o}}$ is given by

$$
v_{\rho}(x)=\frac{\nabla N_{\rho}(x)}{\left|\nabla N_{\rho}(x)\right|} .
$$

In the following, we let $\tau_{\rho}=-\left(\vec{z} \times v_{\rho}\right)$ denote the corresponding tangential vector. Next, suppose that $\rho \in C([0, T], \mathcal{V}) \cap C^{1}\left([0, T], h^{1+\delta}\left(\mathbb{S}^{1}\right)\right)$ for some $T>0$ and set

$$
N_{\rho}(t, x):=N_{\rho(t)}(x)=|x|-1-\rho\left(t, \frac{x}{|x|}\right), \quad t \in[0, T], \quad 3 / 4<|x|<5 / 4 .
$$

Observe that the normal velocity $V_{\rho}$ of the moving boundary $\Gamma_{\rho}$ equals $-\partial_{t} N_{\rho} /\left|\nabla N_{\rho}\right|$. Consequently, if $\rho \in C([0, T], \mathcal{V}) \cap C^{1}\left([0, T], h^{1+\delta}\left(\mathbb{S}^{1}\right)\right)$ describes the evolution of the boundary, then (2.6)-(2.9) read as

$$
\begin{aligned}
\Delta P_{j} & =0 & & \text { in } \Omega_{\rho(t)}^{j}, \quad j=\mathrm{i}, \mathrm{o}, \\
P_{\mathrm{i}}-P_{\mathrm{o}} & =\sigma \kappa_{\Gamma_{\rho(t)}}+\left(\gamma_{\mathrm{o}}-\gamma_{\mathrm{i}}\right)\left[1+\rho\left(t, \frac{x}{|x|}\right)\right]^{2} & & \text { on } \Gamma_{\rho(t)}, \\
\partial_{t} \rho\left(t, \frac{x}{|x|}\right) & =\frac{-1}{\left|\Theta_{j}\right|^{2}}\left[\alpha_{j} \nabla P_{j}+\beta_{j}\left(\vec{z} \times \nabla P_{j}\right)\right] \cdot \nabla N_{\rho(t)} & & \text { on } \Gamma_{\rho(t)}, \quad j=\mathrm{i}, \mathrm{o}, \\
\alpha_{\mathrm{o}} \partial_{\nu} P_{\mathrm{o}}+\beta_{\mathrm{o}} \partial_{\tau} P_{\mathrm{o}} & =0 & & \text { on } \partial \Omega=R \mathbb{S}^{1},
\end{aligned}
$$

for $t>0$ and with $\Gamma_{\rho(0)}=\Gamma^{0}$. We call a triple $\left(\rho, P_{\mathrm{i}}, P_{\mathrm{o}}\right)$ a (classical Hölder) solution to (2.11)(2.14) provided

$$
\rho \in C([0, T], \cup) \cap C^{1}\left([0, T], h^{1+\delta}\left(\mathbb{S}^{1}\right)\right)
$$


for some $T>0$ and

$$
P_{j}(t, \cdot) \in h^{2+\delta}\left(\Omega_{\rho(t)}^{j}\right), \quad t \in[0, T], \quad j=\mathrm{i}, \mathrm{o},
$$

satisfy (2.11)-(2.14) pointwise. The main existence result is stated in the following theorem, a proof of which is given in Section 3:

THEOREM 2.1 Let $\delta \in(0,1)$. There is an open zero neighborhood $\mathcal{O}$ in $h^{4+\delta}\left(\mathbb{S}^{1}\right)$ such that for each initial geometry $\rho_{0} \in \mathcal{O}$, there exists a time $T=T\left(\rho_{0}\right)$ such that a classical solution $\left(\rho, P_{i}, P_{o}\right)$ to (2.11)-(2.14) exists on the interval $[0, T]$ with $\rho(0)=\rho_{0}$. This solution is unique up to a common additive constant in the pressures $P_{\mathrm{i}}$ and $P_{\mathrm{o}}$.

We also investigate stability properties of the trivial solution corresponding to the geometry $\rho=0$, that is, to the unit circle $\mathbb{S}^{1}$, and constant pressures $P_{\mathrm{i}}=c+\sigma+\gamma_{\mathrm{o}}-\gamma_{\mathrm{i}}$ and $P_{\mathrm{o}}=c$ with arbitrary $c \in \mathbb{R}$. The stability properties of the trivial solution are determined by the relative density of the fluids.

THEOREM 2.2 If $\varrho_{\mathrm{o}}>\varrho_{\mathrm{i}}$ then the trivial solution $\left(\rho, P_{\mathrm{i}}, P_{\mathrm{o}}\right)=\left(0, c+\sigma+\gamma_{\mathrm{o}}-\gamma_{\mathrm{i}}, c\right)$ is locally asymptotically stable. However, if $\varrho_{\mathrm{i}}>\varrho_{\mathrm{o}}$, then the trivial solution $\left(\rho, P_{\mathrm{i}}, P_{\mathrm{o}}\right)=\left(0, c+\sigma+\gamma_{\mathrm{o}}-\right.$ $\left.\gamma_{\mathrm{i}}, c\right)$ is unstable.

We refer to Section 4 for more precise statements of the stability results and their proofs. Note that Theorem 2.2 implies that there are no nontrivial equilibrium solutions near the sphere if $\varrho_{\mathrm{o}}>\varrho_{\mathrm{i}}$. Equilibria in general are characterized by the following result:

Proposition 2.3 If $\left(\Gamma, P_{\mathrm{i}}, P_{\mathrm{o}}\right)$ is any equilibrium for (2.6)-(2.9) with $\Gamma \in C^{2+\delta}$ and $P_{j} \in$ $h^{2+\delta}\left(\Omega^{j}\right)$ for $j=\mathrm{i}, \mathrm{o}$, then $P_{j}$ is constant for $j=\mathrm{i}$, o. Moreover, if $\Gamma=\Gamma_{\rho}$ for some $\rho \in V$ and $c:=P_{\mathrm{i}}-P_{\mathrm{o}} \in \mathbb{R}$, then $\rho \in C^{\infty}\left(\mathbb{S}^{1}\right)$ satisfies

$$
c=\gamma \frac{(1+\rho)^{2}+2 \dot{\rho}^{2}-(1+\rho) \ddot{\rho}}{\left[(1+\rho)^{2}+\dot{\rho}^{2}\right]^{3 / 2}}+\left(\zeta_{\mathrm{o}}-\zeta_{\mathrm{i}}\right)(1+\rho)^{2} \quad \text { on } \mathbb{S}^{1} .
$$

This statement is proved in Section 5. Equation (2.15) for the geometry determines equilibria completely. Moreover, the fact that equilibria occur only if the pressures are both constant has a number of implications. In particular, equilibria for the two-phase problem coincide with the equilibria for the one-phase problem (with the fluid inside). More precisely, the one-phase equilibrium problem (as treated, e.g., in [7]) may be regarded as a two-phase equilibrium problem by taking $P_{\mathrm{o}}=0$ and $\varrho_{\mathrm{o}}=0$. Conversely, the two-phase equilibrium problem is equal to the one-phase equilibrium problem by taking $P_{\mathrm{o}}=$ const . Furthermore, as the pressures at equilibria are necessarily constant, Coriolis force has no influence on the existence of equilibria. Therefore, there are no equilibria for (2.6)-(2.9) if $\varrho_{\mathrm{o}} \geqslant \varrho_{\mathrm{i}}$ as noted above (the case $\varrho_{\mathrm{o}}=\varrho_{\mathrm{i}}$ corresponds to considering just one fluid of density zero) while the case $\varrho_{0}<\varrho_{i}$ has been investigated in [7]. The unstable nontrivial equilibria constructed therein ${ }^{3}$ by means of bifurcation theory are also equilibria for (2.6)-(2.9).

\footnotetext{
${ }^{3}$ In [7] the fluid is assumed to have density $\varrho=1$. So to compare it with our results one has to replace $\omega^{2}$ by $\omega^{2} \varrho$ therein.
} 


\section{Proof of Theorem 2.1}

The proof of Theorem 2.1 is best carried out in a coordinate system in which the moving interface between the liquids is fixed. We therefore begin with the transformation to a fixed domain formulation.

\subsection{An equivalent problem on fixed domains}

We transform the free boundary problem to fixed domains using the standard Hanzawa-transform. Let $\varphi: \mathbb{R} \rightarrow[0,1]$ be a smooth function with $\varphi(r)=1$ for $|r| \leqslant a$ and $\varphi(r)=0$ for $|r| \geqslant 3 a$ and $\left\|\varphi^{\prime}\right\|_{\infty}<1 / a$. We introduce a family of $C^{4+\delta}$-diffeomorphisms

$$
\phi_{\rho} \in \operatorname{Diff}^{4+\delta}\left(\mathbb{R}^{2}, \mathbb{R}^{2}\right) \cap \operatorname{Diff}^{4+\delta}\left(B^{\mathrm{i}}, \Omega_{\rho}^{\mathrm{i}}\right) \cap \operatorname{Diff}^{4+\delta}\left(B^{\mathrm{o}}, \Omega_{\rho}^{\mathrm{o}}\right), \quad \rho \in \mathcal{V},
$$

where

$$
B^{\mathrm{i}}:=\mathbb{B}(0,1), \quad B^{\mathrm{o}}:=\Omega \backslash \overline{B^{\mathrm{i}}},
$$

by setting

$$
\phi_{\rho}(x):= \begin{cases}{\left[|x|+\varphi(|x|-1) \rho\left(\frac{x}{|x|}\right)\right] \frac{x}{|x|}} & \text { if } 0<|x|<2, \\ x & \text { else. }\end{cases}
$$

Note that $\phi_{\rho}$ maps $\mathbb{S}^{1}$ onto $\Gamma_{\rho}$. Given $\rho \in \mathcal{V}$, let

$$
\phi_{\rho}^{*}: B U C\left(\Omega_{\rho}^{j}\right) \rightarrow B U C\left(B^{j}\right), \quad p \mapsto p \circ \phi_{\rho}
$$

denote the push-forward operator and

$$
\phi_{*}^{\rho}: B U C\left(B^{j}\right) \rightarrow B U C\left(\Omega_{\rho}^{j}\right), \quad q \mapsto q \circ \phi_{\rho}^{-1}
$$

the pull-back operator induced by $\phi_{\rho}$. Given $\rho \in V$, the transformed differential and boundary operators acting on $Q_{j}:=\phi_{\rho}^{*} P_{j}, j=\mathrm{i}, \mathrm{o}$ are given by

$$
Q_{j}(\rho) Q_{j}:=\phi_{\rho}^{*}\left(\Delta\left(\phi_{*}^{\rho} Q_{j}\right)\right)
$$

and by

$$
\mathbb{B}_{j}(\rho) Q_{j}:=\frac{-1}{\left|\Theta_{j}\right|^{2}}\left[\alpha_{j}\left(\phi_{\rho}^{*} \nabla\left(\phi_{*}^{\rho} Q_{j}\right)\right)+\beta_{j}\left(\vec{z} \times\left(\phi_{\rho}^{*} \nabla\left(\phi_{*}^{\rho} Q_{j}\right)\right)\right)\right] \cdot\left(\phi_{\rho}^{*} \nabla N_{\rho}\right) .
$$

Defining

$$
\mathcal{K}(\rho):=\sigma \phi_{\rho}^{*} \kappa_{\Gamma_{\rho}}+\left(\gamma_{\mathrm{o}}-\gamma_{\mathrm{i}}\right)|1+\rho|^{2}
$$

for $\rho \in V$ and

$$
\mathbb{B}_{0}:=\alpha_{\mathrm{o}} \partial_{\nu}+\beta_{\mathrm{o}} \partial_{\tau} \quad \text { on } \quad \partial \Omega=R \mathbb{S}^{1},
$$

the free boundary problem (2.11)-(2.14) is transformed to the following problem on fixed domains:

$$
\begin{aligned}
Q_{j}(\rho) Q_{j} & =0 & & \text { in } B^{j}, \quad j=\mathrm{i}, \mathrm{o}, \\
Q_{\mathrm{i}}-Q_{\mathrm{o}} & =\mathcal{K}(\rho) & & \text { on } \mathbb{S}^{1}, \\
\partial_{t} \rho & =\mathbb{B}_{j}(\rho) Q_{j} & & \text { on } \mathbb{S}^{1}, \quad j=\mathrm{i}, \mathrm{o}, \\
\mathbb{B}_{\mathrm{o}} Q_{\mathrm{o}} & =0 & & \text { on } R \mathbb{S}^{1} .
\end{aligned}
$$


We call a triple $\left(\rho, Q_{\mathrm{i}}, Q_{\mathrm{o}}\right)$ a (classical Hölder) solution to (3.1)-(3.4) provided

$$
\rho \in C([0, T], \vartheta) \cap C^{1}\left([0, T], h^{1+\delta}\left(\mathbb{S}^{1}\right)\right)
$$

for some $T>0$ and

$$
Q_{j}(t, \cdot) \in h^{2+\delta}\left(B^{j}\right), \quad t \in[0, T], \quad j=\mathrm{i}, \mathrm{o},
$$

satisfy (3.1)-(3.4) pointwise. With this definition, problems (2.11)-(2.14) and (3.1)-(3.4) are equivalent as follows from the next proposition.

Proposition 3.1 Let $Q_{j}=\phi_{\rho}^{*} P_{j}$ and $P_{j}=\phi_{*}^{\rho} Q_{j}$. Then $\left(\rho, P_{\mathrm{i}}, P_{\mathrm{o}}\right)$ is a solution to (2.11)-(2.14) if and only if $\left(\rho, Q_{\mathrm{i}}, Q_{\mathrm{o}}\right)$ is a solution to (3.1)-(3.4).

Proof. Due to the above definitions of the differential operators, we merely need to ascertain that no regularity loss occurs in the process, i.e., that $P_{j} \in h^{2+\delta}\left(\Omega_{\rho}^{j}\right)$ implies $Q_{j}=\phi_{\rho}^{*} P_{j} \in h^{2+\delta}\left(B^{j}\right)$ for $\rho \in V$ and vice versa. This, however, easily follows from the fact that $\phi_{\rho}^{*} P_{j} \in B U C^{4}\left(B^{j}\right)$ when $P_{j} \in B U C^{\infty}\left(\Omega_{\rho}^{j}\right)$ and $\rho \in V \subset h^{4+\delta}\left(\mathbb{S}^{1}\right)$ and the observation that $h^{2+\delta}\left(B^{j}\right)$ coincides with the closure of $B U C^{4}\left(B^{j}\right)$ in $B U C^{2+\delta}\left(B^{j}\right)$.

Next we collect some regularity properties of the operators defined above.

LEMma 3.2 (i) The operator $Q_{j}(\rho) \in \mathcal{L}\left(h^{2+\delta}\left(B^{j}\right), h^{\delta}\left(B^{j}\right)\right)$ is uniformly elliptic and analytic in $\rho \in V$ for $j=\mathrm{i}, \mathrm{o}$.

(ii) The operator $\mathbb{B}_{j}(\rho) \in \mathcal{L}\left(h^{2+\delta}\left(B^{j}\right), h^{1+\delta}\left(\mathbb{S}^{1}\right)\right)$ is analytic in $\rho \in V$ for $j=\mathrm{i}$, o.

Proof. (i) Given $\rho \in V$, uniform ellipticity of $\mathbb{Q}_{j}(\rho)$ is a consequence of its symbol being

$$
a(\rho)(\xi)=\phi_{\rho}^{*}\left(\left|J\left(\phi_{\rho}^{-1}\right) \xi\right|^{2}+\Delta \phi_{\rho}^{-1} \cdot \xi\right), \quad \xi \in \mathbb{R}^{2},
$$

where the Jacobian $J\left(\phi_{\rho}^{-1}\right)$ of $\phi_{\rho}^{-1}$ satisfies $\left|J\left(\phi_{\rho}^{-1}\right) \xi\right|^{2} \geqslant \epsilon|\xi|^{2}$ for some $\epsilon>0$. Analyticity in $\rho$ follows from the analyticity of $\phi_{\rho}$ and of $J\left(\Phi_{\rho}^{-1}\right)=\Phi_{*}^{\rho} J\left(\Phi_{\rho}\right)^{-1}$. For details we refer to [7, § 3.2]. (ii) Note that

$$
\nabla \rho\left(\frac{x}{|x|}\right)=\rho^{\prime}\left(\frac{x}{|x|}\right) \frac{1}{|x|^{2}}\left(\begin{array}{c}
-x_{2} \\
x_{1}
\end{array}\right), \quad x=\left(x_{1}, x_{2}\right) \in \mathbb{R}^{2} \backslash\{0\}, \quad \rho \in C^{1}\left(\mathbb{S}^{1}\right) .
$$

Hence, for $\rho \in V$ and $\phi_{\rho}=\left(\phi_{\rho}^{1}, \phi_{\rho}^{2}\right)$,

$$
\left(\phi_{\rho}^{*} \nabla N_{\rho}\right)(y)=\frac{\phi_{\rho}(y)}{\left|\phi_{\rho}(y)\right|}-\rho^{\prime}\left(\frac{\phi_{\rho}(y)}{\left|\phi_{\rho}(y)\right|}\right) \frac{1}{\left|\phi_{\rho}(y)\right|^{2}}\left(\begin{array}{c}
-\phi_{\rho}^{2}(y) \\
\phi_{\rho}^{1}(y)
\end{array}\right), \quad y \in \mathbb{S}^{1},
$$

showing that $\left[\rho \mapsto \phi_{\rho}^{*} \nabla N_{\rho}\right]: V \rightarrow h^{3+\delta}\left(\mathbb{S}^{1}\right)$ is analytic. The definition of $\mathbb{B}_{j}(\rho)$ entails its analytic dependence on $\rho$ since

$$
\phi_{\rho}^{*} \nabla\left(\phi_{*}^{\rho} Q_{j}\right)=\nabla Q_{j} \phi_{\rho}^{*} J\left(\phi_{\rho}^{-1}\right) .
$$

The curvature operator $\mathcal{K}$ can easily be computed explicitly. 
LEMma 3.3 The operator $\mathcal{K}: \mathcal{V} \rightarrow h^{2+\delta}\left(\mathbb{S}^{1}\right)$ is analytic and given by

$$
\mathcal{K}(\rho)=\sigma \frac{(1+\rho)^{2}+2 \dot{\rho}^{2}-(1+\rho) \ddot{\rho}}{\left[(1+\rho)^{2}+\dot{\rho}^{2}\right]^{3 / 2}}+\left(\gamma_{\mathrm{o}}-\gamma_{\mathrm{i}}\right)(1+\rho)^{2}, \quad \rho \in \mathcal{V},
$$

and

$$
\partial \mathcal{K}(0)[h]=\sigma(-h-\ddot{h})+2\left(\gamma_{\mathrm{o}}-\gamma_{\mathrm{i}}\right) h, \quad h \in h^{4+\delta}\left(\mathbb{S}^{1}\right) .
$$

Proof. If $\rho \in \mathcal{V}$, a parametrization of $\Gamma_{\rho}$ is given by

$$
(1+\rho(\theta)) e^{i \theta}=: x(\theta)+i y(\theta), \quad \theta \in[0,2 \pi] .
$$

Hence the claim is a consequence of the curvature formula

$$
\kappa_{\Gamma_{\rho}}=\frac{\dot{x} \ddot{y}-\ddot{x} \dot{y}}{\left(\dot{x}^{2}+\dot{y}^{2}\right)^{3 / 2}}
$$

and the definition of $\mathcal{K}(\rho)$.

\subsection{Local well-posedness}

In order to establish local existence and uniqueness of solutions, we split (3.1)-(3.4) in three subproblems: two elliptic problems for the transformed pressures and an evolution equation for the geometry. More precisely, given $\rho \in V$ and a function $h$ defined on $\mathbb{S}^{1}$, we shall first look for solutions $Q_{\mathrm{i}}=S(\rho, h)$ to the following elliptic problem on $B^{\mathrm{i}}$ with Neumann type boundary condition

$$
\begin{array}{rlrl}
Q_{\mathrm{i}}(\rho) Q_{\mathrm{i}} & =0 & & \text { in } B^{\mathrm{i}}, \\
\mathbb{B}_{\mathrm{i}}(\rho) Q_{\mathrm{i}} & =h-\frac{\left|\phi_{\rho}^{*}\left(\nabla N_{\rho}\right)\right|}{\left|\Gamma_{\rho}\right|} \int_{\Gamma_{\rho}} \frac{\phi_{*}^{\rho} h}{\left|\nabla N_{\rho}\right|} \mathrm{d} \sigma & & \text { on } \mathbb{S}^{1}, \\
\int_{\mathbb{S} 1} Q_{\mathrm{i}} \mathrm{d} \sigma & =\int_{\mathbb{S}^{1}} h \mathrm{~d} \sigma, &
\end{array}
$$

where $\left|\Gamma_{\rho}\right|$ stands for the length of the closed curve $\Gamma_{\rho}$. In the second step, we shall study the elliptic problem on the annulus $B^{\circ}$ with Dirichlet-Neumann boundary conditions

$$
\begin{aligned}
\mathbb{Q}_{\mathrm{o}}(\rho) Q_{\mathrm{o}}=0 & \text { in } B^{\mathrm{o}}, \\
Q_{\mathrm{o}}=g & \text { on } \mathbb{S}^{1}, \\
\mathbb{B}_{\mathrm{o}} Q_{\mathrm{o}}=0 & \text { on } R \mathbb{S}^{1},
\end{aligned}
$$

with solution $Q_{\mathrm{o}}=T(\rho, g)$ depending on $\rho \in V$ and $g$ on $\mathbb{S}^{1}$. Finally we use the solution operators $S$ and $T$ to derive the evolution equation

$$
\partial_{t} \rho=\mathbb{B}_{0}(\rho) T\left(\rho, S\left(\rho, \partial_{t} \rho\right)-\mathcal{K}(\rho)\right)
$$

for $\rho$. Note that $\partial_{t} \rho$ appears on both sides of (3.12). As (3.6)-(3.7) is a Neumann-type problem, an additional integral term is introduced on the right hand of side (3.7) which makes the mean zero and 
thus ensures solvability of the problem. The third equation (3.8) guarantees uniqueness. Let us point out that, provided we can solve (3.12) for $\rho=\rho(t)$, the particular form of the boundary operator $\mathbb{B}_{\mathrm{o}}(\rho)$ and the properties of $T(\rho, g)$ yield (see (3.44) below)

$$
\int_{\Gamma_{\rho}} \frac{\phi_{*}^{\rho} \partial_{t} \rho}{\left|\nabla N_{\rho}\right|} \mathrm{d} \sigma=0
$$

Thus the integral term in (3.7) vanishes for $h=\partial_{t} \rho$ and we may take

$$
Q_{\mathrm{i}}(t)=S\left(\rho(t), \partial_{t} \rho(t)\right), \quad Q_{\mathrm{o}}(t)=T\left(\rho(t), Q_{\mathrm{i}}(t)-\mathcal{K}(\rho(t))\right)
$$

to obtain a solution for the original equations (3.1)-(3.4). We shall be more specific at the end of this section.

In the following two propositions we study the solvability of the elliptic problems.

Proposition 3.4 Given $\rho \in V$ and $h \in h^{1+\delta}\left(\mathbb{S}^{1}\right)$, (3.6)-(3.8) possesses a unique solution

$$
Q_{\mathrm{i}}=S(\rho, h) \in h^{2+\delta}\left(B^{\mathrm{i}}\right) \text {, }
$$

and the map

$$
[\rho \mapsto S(\rho, \cdot)]: \mathcal{V} \rightarrow \mathcal{L}\left(h^{1+\delta}\left(\mathbb{S}^{1}\right), h^{2+\delta}\left(B^{\mathrm{i}}\right)\right)
$$

is analytic.

To prove this statement on the fixed domain $B^{\mathrm{i}}$ we shall first prove an auxiliary result on the domain $\Omega_{\rho}^{\mathrm{i}}$ :

LEMMA 3.5 Let $\rho \in V$ and let $v=v_{\Gamma_{\rho}}$ and $\tau=\tau_{\Gamma_{\rho}}$ denote the corresponding outer unit normal and unit tangential vectors to $\Gamma_{\rho}$, respectively. Set

$$
\mu:=\frac{-1}{\left|\Theta_{\mathrm{i}}\right|^{2}}\left(\alpha_{\mathrm{i}} v+\beta_{\mathrm{i}} \tau\right)
$$

and define

$$
\mathbb{A}: h^{2+\delta}\left(\Omega_{\rho}^{\mathrm{i}}\right) \rightarrow h^{\delta}\left(\Omega_{\rho}^{\mathrm{i}}\right) \times h^{1+\delta}\left(\Gamma_{\rho}\right), \quad u \mapsto\left(-\Delta u, \partial_{\mu} u\right) .
$$

Then $\operatorname{ker}(\mathbb{A})=\mathbb{R} \cdot \mathbf{1}$ and

$$
\operatorname{im}(\mathbb{A})=\left\{(f, g) \in h^{\delta}\left(\Omega_{\rho}^{\mathrm{i}}\right) \times h^{1+\delta}\left(\Gamma_{\rho}\right) ; \int_{\Omega_{\rho}^{\mathrm{i}}} f \mathrm{~d} x=\frac{\left|\Theta_{\mathrm{i}}\right|^{2}}{\alpha_{\mathrm{i}}} \int_{\Gamma_{\rho}} g \mathrm{~d} \sigma\right\} .
$$

Proof. Note that $\mu$ is nowhere tangential and that $\mathbf{1}$ is an eigenfunction of $\mathbb{A}$. Thus, $\operatorname{ker}(\mathbb{A})=\mathbb{R} \cdot \mathbf{1}$ follows from [2, Thm.12.1] since $\left(-\Delta, \partial_{\mu}\right)$ is regular elliptic. To determine the range of $\mathbb{A}$, fix $p>n /(1-\delta)$ and suppose first that $\mathbb{A} u=(f, g)$, that is,

$$
-\Delta u=f \quad \text { in } \Omega_{\rho}^{\mathrm{i}}, \quad \partial_{\mu} u=g \quad \text { on } \Gamma_{\rho} .
$$

Since $\partial_{\tau} w=(\vec{z} \times \nabla w) \cdot v$ and $\operatorname{div}(\vec{z} \times \nabla w)=0$, we have by Gauss' Theorem

$$
\int_{\Gamma_{\rho}} \partial_{\tau} w \mathrm{~d} \sigma=\int_{\Omega_{\rho}^{\mathrm{i}}} \operatorname{div}(\vec{z} \times \nabla w) \mathrm{d} x=0, \quad w \in W_{p}^{2}\left(\Omega_{\rho}^{\mathrm{i}}\right) .
$$


Therefore,

$$
\int_{\Omega_{\rho}^{\mathrm{i}}} f \mathrm{~d} x=\frac{\left|\Theta_{\mathrm{i}}\right|^{2}}{\alpha_{\mathrm{i}}} \int_{\Gamma_{\rho}}\left(-\frac{\alpha_{\mathrm{i}}}{\left|\Theta_{\mathrm{i}}\right|^{2}} \partial_{\nu} u-\frac{\beta_{\mathrm{i}}}{\left|\Theta_{\mathrm{i}}\right|^{2}} \partial_{\tau} u\right) \mathrm{d} \sigma=\frac{\left|\Theta_{\mathrm{i}}\right|^{2}}{\alpha_{\mathrm{i}}} \int_{\Gamma_{\rho}} g \mathrm{~d} \sigma .
$$

For the reverse inclusion we use a Fredholm argument. By [2, Lem.5.1] there is a coretraction

$$
M \in \mathcal{L}\left(W_{p}^{1-1 / p}\left(\Gamma_{\rho}\right), W_{p}^{2}\left(\Omega_{\rho}^{\mathrm{i}}\right)\right)
$$

for the boundary operator such that $\partial_{\mu} M g=g$ for $g \in W_{p}^{1-1 / p}\left(\Gamma_{\rho}\right)$. Then

$$
R:=\Delta M \in \mathcal{L}\left(W_{p}^{1-1 / p}\left(\Gamma_{\rho}\right), L_{p}\left(\Omega_{\rho}^{\mathrm{i}}\right)\right),
$$

and finding a solution $u \in W_{p}^{2}\left(\Omega_{\rho}^{\mathrm{i}}\right)$ to problem (3.13) for a given $(f, g) \in L_{p}\left(\Omega_{\rho}^{\mathrm{i}}\right) \times W_{p}^{1-1 / p}\left(\Gamma_{\rho}\right)$ is equivalent to finding a solution $v \in W_{p}^{2}\left(\Omega_{\rho}^{\mathrm{i}}\right)$ to

$$
-\Delta v=f+R g \quad \text { in } \Omega_{\rho}^{\mathrm{i}}, \quad \partial_{\mu} v=0 \quad \text { on } \Gamma_{\rho},
$$

and setting $u:=v+M g$. Let

$$
W_{p, \mu}^{2}\left(\Omega_{\rho}^{\mathrm{i}}\right):=\left\{v \in W_{p}^{2}\left(\Omega_{\rho}^{\mathrm{i}}\right) ; \partial_{\mu} v=0 \text { on } \Gamma_{\rho}\right\}
$$

and consider the closed linear operator $T$ on $L_{p}\left(\Omega_{\rho}^{\mathrm{i}}\right)$ given by $T v:=-\Delta v$ for $v \in W_{p, \mu}^{2}\left(\Omega_{\rho}^{\mathrm{i}}\right)$. Then, as above, $\operatorname{ker}(T)=\mathbb{R} \cdot \mathbf{1}$, and $T$ has compact resolvent. Hence $T$ is a Fredholm operator on $L_{p}\left(\Omega_{\rho}^{\mathrm{i}}\right)$ with index zero. Since obviously $W_{p, \mu}^{2}\left(\Omega_{\rho}^{\mathrm{i}}\right)$ is dense in $L_{p}\left(\Omega_{\rho}^{\mathrm{i}}\right)$, we also obtain for its dual $T^{\prime}$ that $\operatorname{ker}\left(T^{\prime}\right)=\mathbb{R} \cdot f^{\prime}$ for some $f^{\prime} \in L_{p^{\prime}}\left(\Omega_{\rho}^{\mathrm{i}}\right)$, where $p^{\prime}$ denotes the dual exponent of $p$. Moreover, $T v=h$ with $h \in L_{p}\left(\Omega_{\rho}^{\mathrm{i}}\right)$ is solvable for $v \in W_{p, \mu}^{2}\left(\Omega_{\rho}^{\mathrm{i}}\right)$ if and only if $\left\langle f^{\prime}, h\right\rangle=0$. However, since (3.14) ensures that

$$
\int_{\Omega_{\rho}^{\mathrm{i}}} \Delta v \mathrm{~d} x=-\frac{\left|\Theta_{\mathrm{i}}\right|^{2}}{\alpha_{\mathrm{i}}} \int_{\Gamma_{\rho}} \partial_{\mu} v \mathrm{~d} \sigma=0, \quad v \in W_{p, \mu}^{2}\left(\Omega_{\rho}^{\mathrm{i}}\right),
$$

we may take $f^{\prime}=\mathbf{1}$, that is, $\operatorname{ker}\left(T^{\prime}\right)=\mathbb{R} \cdot \mathbf{1}$, and $T v=f+R g$ is solvable for $v \in W_{p, \mu}^{2}\left(\Omega_{\rho}^{\mathrm{i}}\right)$ with

$$
(f, g) \in L_{p}\left(\Omega_{\rho}^{\mathrm{i}}\right) \times W_{p}^{1-1 / p}\left(\Gamma_{\rho}\right)
$$

if and only if

$$
0=\int_{\Omega_{\rho}^{\mathrm{i}}}(f+R g) \mathrm{d} x=\int_{\Omega_{\rho}^{\mathrm{i}}} f \mathrm{~d} x-\frac{\left|\Theta_{\mathrm{i}}\right|^{2}}{\alpha_{\mathrm{i}}} \int_{\Gamma_{\rho}} \partial_{\mu} g \mathrm{~d} \sigma,
$$

the last equality being again due to (3.14). Finally, if $u$ is the solution to (3.13) for a given

$$
(f, g) \in h^{\delta}\left(\Omega_{\rho}^{\mathrm{i}}\right) \times h^{1+\delta}\left(\Gamma_{\rho}\right) \subset L_{p}\left(\Omega_{\rho}^{\mathrm{i}}\right) \times W_{p}^{1-1 / p}\left(\Gamma_{\rho}\right)
$$

satisfying (3.16), then $u \in W_{p}^{2}\left(\Omega_{\rho}^{\mathrm{i}}\right) \hookrightarrow C^{1+\delta}\left(\overline{\Omega_{\rho}^{\mathrm{i}}}\right)$ by the choice of $p$. Using Schauder regularity theory [11, Thm.6.15] for

$$
-\Delta \bar{u}=f \quad \text { in } \Omega_{\rho}^{\mathrm{i}}, \quad \bar{u}+\partial_{\mu} \bar{u}=g+u \quad \text { on } \Gamma_{\rho},
$$

then easily gives $u=\bar{u} \in h^{2+\delta}\left(\Omega_{\rho}^{\mathrm{i}}\right)$. This proves the claimed characterization of im(A). 
Proof of Proposition 3.4. For $\rho \in V$ fixed, set

$$
P(\rho) h:=\frac{\left|\phi_{\rho}^{*}\left(\nabla N_{\rho}\right)\right|}{\left|\Gamma_{\rho}\right|} \int_{\Gamma_{\rho}} \frac{\phi_{*}^{\rho} h}{\left|\nabla N_{\rho}\right|} \mathrm{d} \sigma, \quad h \in h^{1+\delta}\left(\mathbb{S}^{1}\right),
$$

and define an operator $\mathbb{A}_{\mathrm{i}}(\rho): h^{2+\delta}\left(B^{\mathrm{i}}\right) \rightarrow h^{\delta}\left(B^{\mathrm{i}}\right) \times h^{1+\delta}\left(\mathbb{S}^{1}\right)$ by

$$
\mathbb{A}_{\mathrm{i}}(\rho) u:=\left(D(\rho) \mathbb{Q}_{\mathrm{i}}(\rho) u, d(\rho) \mathbb{B}_{\mathrm{i}}(\rho) u\right), \quad u \in h^{2+\delta}\left(B^{\mathrm{i}}\right),
$$

where $D(\rho):=\left|\operatorname{det} \partial_{x} \phi_{\rho}\right|$ and $d(\rho):=1+\rho$. Then, since $D(\rho)>0$ and $d(\rho)>0$, we readily obtain from Lemma 3.5 that $\operatorname{ker}\left(\mathbb{A}_{\mathrm{i}}(\rho)\right)=\mathbb{R} \cdot \mathbf{1}$. Moreover, $(f, g) \in \operatorname{im}\left(\mathbb{A}_{\mathrm{i}}(\rho)\right)$ if and only if $(\hat{f}, \hat{g}) \in \operatorname{im}(\mathbb{A})$, where

$$
\hat{f}:=\phi_{*}^{\rho} \frac{f}{D(\rho)} \in h^{\delta}\left(\Omega_{\rho}^{\mathrm{i}}\right), \quad \hat{g}:=\frac{1}{\left|\nabla N_{\rho}\right|} \phi_{*}^{\rho} \frac{g}{d(\rho)} \in h^{1+\delta}\left(\Gamma_{\rho}\right) .
$$

By virtue of (3.5) we have that

$$
\left|\nabla N_{\rho}\right|=\frac{\sqrt{\dot{\rho}+(1+\rho)^{2}}}{1+\rho} \text { on } \mathbb{S}^{1},
$$

and we infer from Lemma 3.5 that

$$
\operatorname{im}\left(\mathbb{A}_{\mathrm{i}}(\rho)\right)=\left\{(f, g) \in h^{\delta}\left(B^{\mathrm{i}}\right) \times h^{1+\delta}\left(\mathbb{S}^{1}\right) ; \int_{B^{\mathrm{i}}} f \mathrm{~d} x=\frac{\left|\Theta_{\mathrm{i}}\right|^{2}}{\alpha_{\mathrm{i}}} \int_{\mathbb{S}^{1}} g \mathrm{~d} \sigma\right\}=: Y
$$

which is independent of $\rho$. Consequently, the map

$$
\mathbb{A}_{\mathrm{i}}(\rho): h^{2+\delta}\left(B^{\mathrm{i}}\right) /_{\mathbb{R} \cdot \mathbf{1}} \longrightarrow Y
$$

is an isomorphism. Furthermore, $v=\eta(\rho)(f, g):=\mathbb{A}_{\mathrm{i}}(\rho)^{-1}(f, g)$ is a unique solution in $h^{2+\delta}\left(B^{\mathrm{i}}\right) / \mathbb{R} \cdot \mathbf{1}$ to

$$
D(\rho) \mathbb{Q}_{\mathrm{i}}(\rho) v=f \quad \text { on } B^{\mathrm{i}}, \quad d(\rho) \mathbb{B}_{\mathrm{i}}(\rho) v=g \quad \text { on } \mathbb{S}^{1}
$$

for each $\rho \in V$ and $(f, g) \in Y$. As $D(\rho)$ and $d(\rho)$ are analytic in $\rho$, we deduce that

$$
n: v \rightarrow \mathcal{L}\left(Y, h^{2+\delta}\left(B^{\mathrm{i}}\right) / \mathbb{R} \cdot \mathbf{1}\right)
$$

is analytic. Now, we easily check with the help of (3.17) that

$$
\int_{\mathbb{S} 1} d(\rho)(1-P(\rho)) h \mathrm{~d} \sigma=0,
$$

whence $(0, d(\rho)(1-P(\rho)) h) \in Y$ for $h \in h^{1+\delta}\left(\mathbb{S}^{1}\right)$. Thus $u:=\eta(\rho)(0, d(\rho)(1-P(\rho)) h)$ is the unique solution in $h^{2+\delta}\left(B^{\mathrm{i}}\right) /{ }_{\mathbb{R} \cdot \mathbf{1}}$ to

$$
\mathbb{Q}_{\mathrm{i}}(\rho) u=0 \quad \text { on } B^{\mathrm{i}}, \quad \mathbb{B}_{\mathrm{i}}(\rho) u=h-P(\rho) h \quad \text { on } \mathbb{S}^{1} .
$$


By (3.17) we have

$$
P(\rho) h=\frac{\sqrt{\dot{\rho}+(1+\rho)^{2}}}{(1+\rho) \int_{0}^{2 \pi} \sqrt{\dot{\rho}+(1+\rho)^{2}} \mathrm{~d} \theta} \int_{0}^{2 \pi} h(\theta)(1+\rho(\theta)) \mathrm{d} \theta,
$$

hence $P(\rho)$ depends analytically on $\rho$ and so does $u=u(\rho)$. Given $\rho \in V$ and $h \in h^{1+\delta}\left(\mathbb{S}^{1}\right)$, problem (3.6)-(3.8) thus admits a unique solution $Q_{\mathrm{i}}=S(\rho, h)$ and

$$
[\rho \mapsto S(\rho, \cdot)]: \mathcal{V} \rightarrow \mathcal{L}\left(h^{1+\delta}\left(\mathbb{S}^{1}\right), h^{2+\delta}\left(B^{\mathrm{i}}\right)\right)
$$

is analytic. This proves Proposition 3.4.

Proposition 3.6 Given $\rho \in V$ and $g \in h^{2+\delta}\left(\mathbb{S}^{1}\right)$, there is a unique solution $Q_{\mathrm{o}}=T(\rho, g) \in$ $h^{2+\delta}\left(B^{\circ}\right)$ to (3.9)-(3.10). Moreover, the map $[\rho \mapsto T(\rho, \cdot)]: V \rightarrow \mathcal{L}\left(h^{2+\delta}\left(\mathbb{S}^{1}\right), h^{2+\delta}\left(B^{\circ}\right)\right)$ is analytic.

Proof. Let $\rho \in \mathcal{V}$ and define an operator $\mathbb{A}_{0}(\rho): h^{2+\delta}\left(B^{\circ}\right) \rightarrow h^{\delta}\left(B^{\circ}\right) \times h^{2+\delta}\left(\mathbb{S}^{1}\right) \times h^{1+\delta}\left(R \mathbb{S}^{1}\right)$ by

$$
\mathbb{A}_{\mathrm{o}}(\rho) u:=\left(\mathbb{Q}_{\mathrm{o}}(\rho) u,\left.u\right|_{\mathbb{S}^{1}}, \mathbb{B}_{\mathrm{o}} u\right), \quad u \in h^{2+\delta}\left(B^{\mathrm{o}}\right) .
$$

Then $\mathbb{A}_{\mathrm{o}}(\rho)$ is invertible. Indeed, to check its injectivity, let $\mathbb{A}_{\mathrm{o}}(\rho) u=0$. Then, for $\bar{u}:=\phi_{*}^{\rho} u$,

$$
\begin{aligned}
\Delta \bar{u}=0 & \text { in } \quad \Omega_{\rho}^{\mathrm{o},} \\
\bar{u}=0 & \text { on } \quad \Gamma_{\rho}, \\
\alpha_{\mathrm{o}} \partial_{\nu} \bar{u}+\beta_{\mathrm{o}} \partial_{\tau} \bar{u}=0 & \text { on } \quad R \mathbb{S}^{1},
\end{aligned}
$$

and Green's formula yields

$$
\begin{aligned}
0 & =\int_{\Omega_{\rho}^{\mathrm{o}}} \bar{u} \Delta \bar{u} \mathrm{~d} x=-\int_{\Omega_{\rho}^{\mathrm{o}}}|\nabla \bar{u}|^{2} \mathrm{~d} x+\int_{\Gamma_{\rho} \cup R \mathbb{S}^{1}} \bar{u} \partial_{\nu} \bar{u} \mathrm{~d} \sigma \\
& =-\int_{\Omega_{\rho}^{\mathrm{o}}}|\nabla \bar{u}|^{2} \mathrm{~d} x-\frac{\beta_{\mathrm{o}}}{\alpha_{\mathrm{o}}} \int_{\Gamma_{\rho} \cup R \mathbb{S}^{1}} \frac{1}{2} \partial_{\tau} \bar{u}^{2} \mathrm{~d} \sigma \\
& =-\int_{\Omega_{\rho}^{\mathrm{o}}}|\nabla \bar{u}|^{2} \mathrm{~d} x-\frac{\beta_{\mathrm{o}}}{2 \alpha_{\mathrm{o}}} \int_{\Omega_{\rho}^{\mathrm{o}}} \operatorname{div}\left(\begin{array}{c}
-\partial_{2} \bar{u}^{2} \\
\partial_{1} \bar{u}^{2}
\end{array}\right) \mathrm{d} x=-\int_{\Omega_{\rho}^{\mathrm{o}}}|\nabla \bar{u}|^{2} \mathrm{~d} x .
\end{aligned}
$$

This entails $\bar{u}=0$, i.e. $u=0$. To determine the range of $\mathbb{A}_{\mathrm{o}}(\rho)$ consider again the transformed problem

$$
\begin{aligned}
\Delta \bar{u}=\bar{f} & \text { in } \quad \Omega_{\rho}^{\mathrm{o}}, \\
\bar{u}=\bar{g} & \text { on } \quad \Gamma_{\rho}, \\
\alpha_{\mathrm{o}} \partial_{\nu} \bar{u}+\beta_{\mathrm{o}} \partial_{\tau} \bar{u}=\bar{h} & \text { on } \quad R \mathbb{S}^{1} .
\end{aligned}
$$

By means of a coretraction as in the proof of Lemma 3.5 we may assume $\bar{g}=0$ and $\bar{h}=0$. However, by [2],

$$
\lambda-\Delta:\left\{v \in W_{2}^{2}\left(\Omega_{\rho}^{\mathrm{o}}\right) ; v=0 \text { on } \Gamma_{\rho},\left(\alpha_{\mathrm{o}} \partial_{v}+\beta_{\mathrm{o}} \partial_{\tau}\right) v=0 \text { on } R \mathbb{S}^{1}\right\} \longrightarrow L_{2}\left(\Omega_{\rho}^{\mathrm{o}}\right)
$$


is invertible for $\lambda \geqslant 0$ sufficiently large, and the same argument as used to prove its injectivity implies that we actually may take $\lambda=0$. Thus, (3.18)-(3.20) is uniquely solvable for each $\bar{f} \in$ $L_{2}\left(\Omega_{\rho}^{\circ}\right), \bar{g} \in H^{3 / 2}\left(\Gamma_{\rho}\right)$, and $\bar{h} \in H^{1 / 2}\left(R \mathbb{S}^{1}\right)$. Schauder regularity theory ensures $\bar{u} \in h^{2+\delta}\left(\Omega_{\rho}^{\mathrm{o}}\right)$ provided $\bar{f} \in h^{\delta}\left(\Omega_{\rho}^{\mathrm{o}}\right), \bar{g} \in h^{2+\delta}\left(\Gamma_{\rho}\right)$, and $\bar{h} \in h^{1+\delta}\left(R \mathbb{S}^{1}\right)$. Consequently, $\mathbb{A}_{\mathrm{o}}(\rho)$ is surjective and thus invertible, and the claim on the analyticity of $T(\rho, \cdot)=\mathbb{A}_{\mathrm{o}}(\rho)^{-1}(0, \cdot, 0)$ follows from Lemma 3.2.

Next, we state a multiplier result that we shall use later. The proof is a straightforward modification of the case $r=s$ in [3].

Proposition 3.7 Let $r, s \in(0, \infty) \backslash \mathbb{N}$ and $\left(M_{n}\right)_{n \in \mathbb{Z}}$ be a sequence in $\mathbb{C}$ satisfying

(i) $\sup _{n \in \mathbb{Z} \backslash\{0\}}|n|^{r-s}\left|M_{n}\right|<\infty$,

(ii) $\sup _{n \in \mathbb{Z} \backslash\{0\}}|n|^{r-s+1}\left|M_{n+1}-M_{n}\right|<\infty$,

(iii) $\sup _{n \in \mathbb{Z} \backslash\{0\}}|n|^{r-s+2}\left|M_{n+2}-2 M_{n+1}+M_{n}\right|<\infty$.

Then the mapping

$$
\sum_{n \in \mathbb{Z}} \hat{h}_{n} e^{i n \theta} \longmapsto \sum_{n \in \mathbb{Z}} \hat{h}_{n} M_{n} e^{i n \theta}
$$

belongs to $\mathcal{L}\left(C^{s}\left(\mathbb{S}^{1}\right), C^{r}\left(\mathbb{S}^{1}\right)\right)$.

We now focus on the evolution equation (3.12), which we may rewrite, using linearity of the solution operators $S$ and $T$, as

$$
(1-\mathbb{R}(\rho)) \partial_{t} \rho=\mathbb{B}_{\mathrm{o}}(\rho) T(\rho,-\mathcal{K}(\rho))
$$

with

$$
\mathbb{R}(\rho) z:=\mathbb{B}_{\mathrm{o}}(\rho) T(\rho, S(\rho, z)), \quad z \in h^{1+\delta}\left(\mathbb{S}^{1}\right), \quad \rho \in V .
$$

To solve (3.21) for $\partial_{t} \rho$, we use the following

LEMMA 3.8 There is an open zero neighborhood $w \subset v$ in $h^{4+\delta}\left(\mathbb{S}^{1}\right)$ such that $1-\mathbb{R}(\rho)$ is an isomorphism on $h^{1+\delta}\left(\mathbb{S}^{1}\right)$ for each $\rho \in W$.

Proof. By the smooth dependence on $\rho$ stated in Lemma 3.3, Proposition 3.4, and Proposition 3.6, it suffices to prove that $1-\mathbb{R}(0) \in \mathcal{L}\left(h^{1+\delta}\left(\mathbb{S}^{1}\right)\right)$ is invertible. To do this we derive its Fourier expansion in polar coordinates $(r, \theta)$. To compute $Q_{\mathrm{i}}=S(0, h)$ we first note that, for $\rho=0$, problem (3.6)-(3.8) becomes

$$
\begin{array}{rlrl}
\left(\frac{1}{r} \partial_{r}\left(r \partial_{r}\right)+\frac{1}{r^{2}} \partial_{\theta}^{2}\right) Q_{\mathrm{i}} & =0 & \text { in }[r<1], \\
\frac{-1}{\left|\Theta_{\mathrm{i}}\right|}\left(\alpha_{\mathrm{i}} \partial_{r}-\beta_{\mathrm{i}} \partial_{\theta}\right) Q_{\mathrm{i}} & =h-\frac{1}{2 \pi} \int_{0}^{2 \pi} h(\theta) \mathrm{d} \theta & & \text { on } \quad[r=1], \\
\int_{0}^{2 \pi} Q_{\mathrm{i}}(1, \theta) \mathrm{d} \theta & =\int_{0}^{2 \pi} h(\theta) \mathrm{d} \theta, &
\end{array}
$$

which, for a given $h \in h^{1+\delta}\left(\mathbb{S}^{1}\right)$ with expansion

$$
h(\theta)=\sum_{n \in \mathbb{Z}} \hat{h}_{n} e^{i n \theta},
$$


has the unique solution

$$
Q_{\mathrm{i}}(r, \theta)=\hat{h}_{0}+\sum_{n \in \mathbb{Z} \backslash\{0\}} \hat{h}_{n} \frac{\left|\Theta_{\mathrm{i}}\right|^{2}}{-|n| \alpha_{\mathrm{i}}+i n \beta_{\mathrm{i}}} r^{|n|} e^{i n \theta} .
$$

Similarly, for $\rho=0$, problem (3.9)-(3.10) reads as

$$
\begin{aligned}
\left(\frac{1}{r} \partial_{r}\left(r \partial_{r}\right)+\frac{1}{r^{2}} \partial_{\theta}^{2}\right) Q_{\mathrm{o}} & =0 & & \text { in } \quad[1<r<R], \\
Q_{\mathrm{o}} & =g & & \text { on } \quad[r=1], \\
\left(\alpha_{\mathrm{o}} \partial_{r}-\frac{\beta_{\mathrm{o}}}{R} \partial_{\theta}\right) Q_{\mathrm{o}} & =0 & & \text { on } \quad[r=R],
\end{aligned}
$$

and for $g \in h^{2+\delta}\left(\mathbb{S}^{1}\right)$ with expansion

$$
g(\theta)=\sum_{n \in \mathbb{Z}} \hat{g}_{n} e^{i n \theta}
$$

the unique solution $Q_{\mathrm{o}}=T(0, g)$ is given by

$$
Q_{\mathrm{o}}(r, \theta)=\hat{g}_{0}+\sum_{n \in \mathbb{Z} \backslash\{0\}} \hat{g}_{n}\left(\frac{\Theta_{\mathrm{o}}}{\Theta_{\mathrm{o}}+R^{2 n} \bar{\Theta}_{\mathrm{o}}} r^{n}+\frac{\bar{\Theta}_{\mathrm{o}}}{\bar{\Theta}_{\mathrm{o}}+R^{-2 n} \Theta_{\mathrm{o}}} r^{-n}\right) e^{i n \theta}
$$

Therefore, given $h \in h^{1+\delta}\left(\mathbb{S}^{1}\right)$ with expansion (3.22), we have

$$
R(0) h=\frac{-1}{\left|\Theta_{\mathrm{o}}\right|}\left(\alpha_{\mathrm{o}} \partial_{r}-\beta_{\mathrm{o}} \partial_{\theta}\right) T(0, S(0, h))=\sum_{n \in \mathbb{Z} \backslash\{0\}} \hat{h}_{n} l_{n} e^{i n \theta},
$$

where

$$
l_{n}:=\frac{\left(1-R^{2 n}\right)\left|\Theta_{\mathrm{i}}\right|^{2}}{\left(\operatorname{sign}(n) \alpha_{\mathrm{i}}-i \beta_{\mathrm{i}}\right)\left(\Theta_{\mathrm{o}}+R^{2 n} \bar{\Theta}_{\mathrm{o}}\right)}, \quad n \in \mathbb{Z} \backslash\{0\} .
$$

We next use Proposition 3.7 with $M_{n}:=\left(1-l_{n}\right)^{-1}$ to check that $1-\mathbb{R}(0)$ is invertible on $C^{1+\delta}\left(\mathbb{S}^{1}\right)$. Note that $l_{n} \neq 1$ for each $n \in \mathbb{Z} \backslash\{0\}$ since $\alpha_{j}>0, j=\mathrm{i}$, o. Also,

$$
\lim _{n \rightarrow \infty} l_{n}=-\frac{\Theta_{\mathrm{i}} \Theta_{\mathrm{o}}}{\left|\Theta_{\mathrm{o}}\right|^{2}} \neq 1, \quad \lim _{n \rightarrow-\infty} l_{n}=-\frac{\bar{\Theta}_{\mathrm{i}} \bar{\Theta}_{\mathrm{o}}}{\left|\Theta_{\mathrm{o}}\right|^{2}} \neq 1,
$$

so that

$$
\sup _{n \in \mathbb{Z} \backslash\{0\}}\left|M_{n}\right|<\infty
$$

Next,

$$
M_{n+1}-M_{n}=\frac{l_{n+1}-l_{n}}{\left(1-l_{n+1}\right)\left(1-l_{n}\right)},
$$

where, for $n \geqslant 1$,

$$
l_{n+1}-l_{n}=\frac{O\left(R^{2 n+2}\right)}{\bar{\Theta}_{\mathrm{o}}^{2} R^{4 n+2}+O\left(R^{2 n+2}\right)}, \quad n \geqslant 1, \quad l_{n+1}-l_{n}=\frac{O\left(R^{2 n}\right)}{\bar{\Theta}_{\mathrm{o}}^{2}+O\left(R^{2 n}\right)}, \quad n \leqslant-1 .
$$


Since $n R^{-2 n} \rightarrow 0$ as $n \rightarrow \infty$ and $n R^{2 n} \rightarrow 0$ as $n \rightarrow-\infty$, it follows from (3.27) that

$$
\sup _{n \in \mathbb{Z} \backslash\{0\}}|n|\left|M_{n+1}-M_{n}\right|<\infty .
$$

Finally, as above, we obtain from (3.26) and (3.27) that

$$
\begin{aligned}
n^{2} \mid M_{n+2}-2 M_{n+1} & +M_{n}\left|=n^{2}\right| \frac{l_{n+2}-2 l_{n+1}+l_{n}+l_{n}\left(l_{n+1}-l_{n+2}\right)+l_{n+2}\left(l_{n+1}-l_{n}\right)}{\left(1-l_{n+2}\right)\left(1-l_{n+1}\right)\left(1-l_{n}\right)} \mid \\
& \leqslant\left(\sup _{n}\left|M_{n}\right|\right)^{3}\left[\left(1+\left|l_{n}\right|\right) n^{2}\left|l_{n+2}-l_{n+1}\right|+\left(1+\left|l_{n+2}\right|\right) n^{2}\left|l_{n+1}-l_{n}\right|\right] \\
& \leqslant c \sup _{n}\left(n^{2}\left|l_{n+1}-l_{n}\right|\right)<\infty
\end{aligned}
$$

Consequently, Lemma 3.7 implies $(1-\mathbb{R}(0))^{-1} \in \mathcal{L}\left(C^{1+\delta}\left(\mathbb{S}^{1}\right)\right)$. But then $(1-\mathbb{R}(0))^{-1}$ is also a bounded operator on

$$
H^{s}\left(\mathbb{S}^{1}\right)=\left\{h \in L_{2}\left(\mathbb{S}^{1}\right) ;\|h\|_{H^{s}}:=\sum_{n \in \mathbb{Z}}\left(1+n^{2}\right)^{s}\left|\hat{h}_{n}\right|^{2}<\infty\right\}
$$

for each $s>0$ due to (3.27). Recalling that $H^{s}\left(\mathbb{S}^{1}\right)$ is densely embedded in $h^{1+\delta}\left(\mathbb{S}^{1}\right)$ provided $s>5 / 2$, we deduce that $(1-\mathbb{R}(0))^{-1} \in \mathcal{L}\left(h^{1+\delta}\left(\mathbb{S}^{1}\right)\right)$.

According to Lemma 3.8 and (3.21), we are led to look for solutions

$$
\rho \in C([0, T], w) \cap C^{1}\left([0, T], h^{1+\delta}\left(\mathbb{S}^{1}\right)\right)
$$

to the fully nonlinear equation

$$
\partial_{t} \rho=(1-\mathbb{R}(\rho))^{-1} \mathbb{B}_{\mathrm{o}}(\rho) T(\rho,-\mathcal{K}(\rho))=: F(\rho), \quad t \in(0, T] .
$$

The following proposition is instrumental in the computation of the linearization in zero of this evolution equation.

Proposition $3.9 F \in C^{\infty}\left(W, h^{1+\delta}\left(\mathbb{S}^{1}\right)\right)$ and, for $h \in h^{4+\delta}\left(\mathbb{S}^{1}\right)$,

$$
\partial F(0)[h]=(1-R(0))^{-1} B_{\mathrm{o}}(0) T\left(0,\left(2\left(\gamma_{\mathrm{i}}-\gamma_{\mathrm{o}}\right)+\sigma\right) h+\sigma \ddot{h}\right) .
$$

In particular, if $h \in h^{4+\delta}\left(\mathbb{S}^{1}\right)$ with $h(\theta)=\sum_{n \in \mathbb{Z}} \hat{h}_{n} e^{i n \theta}$, then

$$
\partial F(0)[h](\theta)=\sum_{n \in \mathbb{Z} \backslash\{0\}} \hat{h}_{n} q_{n} e^{i n \theta}
$$

where, for $n \in \mathbb{Z} \backslash\{0\}$,

$$
q_{n}:=\frac{A_{n}+i \operatorname{sign}(n) B}{A_{n}^{2}+B^{2}} \mu(n)
$$

with

$$
A_{n}:=\operatorname{sign}(n) \frac{R^{2 n}+1}{R^{2 n}-1} \alpha_{\mathrm{o}}+\alpha_{\mathrm{i}}, \quad B:=\beta_{\mathrm{o}}-\beta_{\mathrm{i}}, \quad \mu(n):=|n|\left(\sigma-2\left(\gamma_{\mathrm{o}}-\gamma_{\mathrm{i}}\right)-\sigma n^{2}\right) .
$$


Proof. Smoothness of the map $F$ follows from Lemma 3.3, Proposition 3.4, Proposition 3.6, and Lemma 3.8. Let $h \in h^{4+\delta}\left(\mathbb{S}^{1}\right)$. Then

$$
\begin{aligned}
\partial F(0)[h]= & \partial(1-\mathbb{R}(\cdot))^{-1}(0)[h] \mathbb{B}_{0}(0) T(0,-\mathcal{K}(0))+(1-\mathbb{R}(0))^{-1} \partial \mathbb{B}_{0}(0)[h] T(0,-\mathcal{K}(0)) \\
& +(1-\mathbb{R}(0))^{-1} \mathbb{B}_{0}(0) \partial_{\rho} T(0,-\mathcal{K}(0))[h]+(1-\mathbb{R}(0))^{-1} \mathbb{B}_{0}(0) T(0,-\partial \mathcal{K}(0)[h]) .
\end{aligned}
$$

But $-\mathcal{K}(0)=-\sigma-\gamma_{\mathrm{o}}+\gamma_{\mathrm{i}}=: c \in \mathbb{R}$ and $T(0, c)=c$ by uniqueness, so $\partial \mathbb{B}_{0}(0)[h]=0$ as this is the derivative of $\left(\rho \mapsto \mathbb{B}_{0}(\rho) c=0\right)$ and similarly $\partial_{\rho} T(0,-\mathcal{K}(0))[h]=0$ as this is the derivative of $(\rho \mapsto T(\rho, c)=c)$. The formula for $\partial F(0)[h]$ now follows from Lemma 3.3. To compute its Fourier expansion, consider $h \in h^{4+\delta}\left(\mathbb{S}^{1}\right)$ with $h(\theta)=\sum_{n \in \mathbb{Z}} \hat{h}_{n} e^{i n \theta}$. Invoking (3.24) and recalling that $\mathbb{B}_{0}(0)=-\left(\alpha_{\mathrm{o}} \partial_{r}-\beta_{\mathrm{o}} \partial_{\theta}\right) /\left|\Theta_{\mathrm{o}}\right|^{2}$ on $\mathbb{S}^{1}$, we obtain

$$
\begin{aligned}
\partial F(0)[h](\theta) & =(1-\mathbb{R}(0))^{-1} \mathbb{B}_{\mathrm{o}}(0) T\left(0,\left(2\left(\gamma_{\mathrm{i}}-\gamma_{\mathrm{o}}\right)+\sigma\right) h+\sigma \ddot{h}\right)(\theta) \\
& =\sum_{n \in \mathbb{Z} \backslash\{0\}} \hat{h}_{n} \frac{n\left(2\left(\gamma_{\mathrm{i}}-\gamma_{\mathrm{o}}\right)+\sigma-\sigma n^{2}\right)\left(R^{2 n}-1\right)}{\Theta_{\mathrm{o}}+R^{2 n} \bar{\Theta}_{\mathrm{o}}} \frac{1}{1-l_{n}} e^{i n \theta}
\end{aligned}
$$

with $l_{n}$ given by (3.25). Elementary calculations now lead to the assertion.

Observe that $A_{n}=A_{-n}$ for $n \in \mathbb{Z} \backslash\{0\}$ and that

$$
A_{n} \searrow \alpha_{\mathrm{o}}+\alpha_{\mathrm{i}} \text { as } \quad|n| \rightarrow \infty .
$$

The next proposition is a consequence of the previous lemma and fundamental for our wellposedness result.

Proposition $3.10-\partial F(0) \in \mathcal{H}\left(h^{4+\delta}\left(\mathbb{S}^{1}\right), h^{1+\delta}\left(\mathbb{S}^{1}\right)\right)$, that is, $\partial F(0) \in \mathcal{L}\left(h^{4+\delta}\left(\mathbb{S}^{1}\right), h^{1+\delta}\left(\mathbb{S}^{1}\right)\right)$ is the generator of an analytic semigroup on $h^{1+\delta}\left(\mathbb{S}^{1}\right)$.

Proof. Based on Proposition 3.7, we prove in a first step that $(\lambda-\partial F(0))^{-1} \in$ $\mathcal{L}\left(h^{1+\delta}\left(\mathbb{S}^{1}\right), h^{4+\delta}\left(\mathbb{S}^{1}\right)\right)$, where, according to Proposition 3.9 (with $q_{0}:=0$ ), we have that

$$
(\lambda-\partial F(0))^{-1} h=\sum_{n \in \mathbb{Z}} \hat{h}_{n} \frac{1}{\lambda-q_{n}} e^{i n \theta}, \quad \operatorname{Re} \lambda \geqslant \lambda_{*},
$$

for $h(\theta)=\sum_{n \in \mathbb{Z}} \hat{h}_{n} e^{i n \theta}$ and, by (3.30),

$$
\lambda_{*}:=1+\frac{2\left|\gamma_{\mathrm{o}}-\gamma_{\mathrm{i}}\right|}{\alpha_{\mathrm{o}}+\alpha_{\mathrm{i}}}>\operatorname{Re} q_{n}, \quad n \in \mathbb{Z} .
$$

Let $\lambda \in \mathbb{C}$ with $\operatorname{Re} \lambda \geqslant \lambda_{*}$ be fixed and set $Q_{n}^{\lambda}:=\left(\lambda-q_{n}\right)^{-1}$. Then

$$
\sup _{n \in \mathbb{Z} \backslash\{0\}}|n|^{3}\left|Q_{n}^{\lambda}\right|<\infty,
$$

since

$$
\frac{q_{n}}{n^{3}} \longrightarrow \pm \frac{\alpha_{\mathrm{o}}+\alpha_{\mathrm{i}} \pm i B}{\left(\alpha_{\mathrm{o}}+\alpha_{\mathrm{i}}\right)^{2}+B^{2}} \sigma \quad \text { as } \quad n \longrightarrow \pm \infty
$$


Next observe that, for each $m \in \mathbb{N}$,

$$
|n|^{m}\left|A_{n+1}-A_{n}\right|=|n|^{m} \frac{2 R^{2 n}(R-1)}{\left(R^{2 n}-1\right)\left(R^{2 n+1}-1\right)} \longrightarrow 0 \quad \text { as } \quad n \longrightarrow \pm \infty .
$$

Thus, letting $z_{n}:=q_{n} / \mu(n)$ we derive from (3.30)

$$
|n|^{m}\left|z_{n+1}-z_{n}\right| \leqslant \frac{A_{n+1} A_{n}}{\left(A_{n+1}^{2}+B^{2}\right)\left(A_{n}^{2}+B^{2}\right)}|n|^{m}\left|A_{n+1}-A_{n}\right|+|n|^{m}\left|A_{n+1}-A_{n}\right| B^{2},
$$

so, for each $m \in \mathbb{N}$,

$$
|n|^{m}\left|z_{n+1}-z_{n}\right| \longrightarrow 0 \quad \text { as } \quad|n| \longrightarrow \infty
$$

Thus, (3.31) gives

$$
\begin{aligned}
|n|^{4}\left|Q_{n+1}^{\lambda}-Q_{n}^{\lambda}\right| & =\left|n^{3} Q_{n+1}^{\lambda}\right|\left|n^{3} Q_{n}^{\lambda}\right|\left|\frac{q_{n+1}-q_{n}}{n^{2}}\right| \\
& \leqslant c \frac{\left|z_{n+1}\right||\mu(n+1)-\mu(n)|}{n^{2}}+c\left|z_{n+1}-z_{n}\right||n|\left|\frac{\mu(n)}{n^{3}}\right| .
\end{aligned}
$$

Taking into account that $\left(z_{n}\right)$ is bounded and that $\left|\mu(n) / n^{3}\right| \rightarrow \sigma$ as $|n| \rightarrow \infty$, we conclude

$$
\sup _{n \in \mathbb{Z} \backslash\{0\}}|n|^{4}\left|Q_{n+1}^{\lambda}-Q_{n}^{\lambda}\right|<\infty
$$

In particular, we have shown

$$
\sup _{n \in \mathbb{Z} \backslash\{0\}} n^{-2}\left|q_{n+1}-q_{n}\right|<\infty
$$

Observe then that

$$
\mu(n)[\mu(n+1)-\mu(n+2)]+\mu(n+2)[\mu(n+1)-\mu(n)]=O\left(n^{4}\right),
$$

hence, due to (3.32),

$$
\begin{aligned}
& \left|\frac{1}{n^{4}}\left[q_{n}\left(q_{n+1}-q_{n+2}\right)+q_{n+2}\left(q_{n+1}-q_{n}\right)\right]\right| \\
& \leqslant\left|z_{n} z_{n+1} \frac{\mu(n)[\mu(n+1)-\mu(n+2)]+\mu(n+2)[\mu(n+1)-\mu(n)]}{n^{4}}\right| \\
& \quad+\left|z_{n} \frac{\mu(n)}{n^{3}} \frac{\mu(n+1)}{n^{3}}\left(z_{n+1}-z_{n+2}\right) n^{2}\right|+\left|z_{n} \frac{\mu(n)}{n^{3}} \frac{\mu(n+2)}{n^{3}}\left(z_{n+1}-z_{n+2}\right) n^{2}\right| \\
& \quad+\left|z_{n+1} \frac{\mu(n+2)}{n^{3}} \frac{\mu(n+1)}{n^{3}}\left(z_{n+2}-z_{n}\right) n^{2}\right| \\
& \leqslant c .
\end{aligned}
$$


Writing

$$
\begin{aligned}
|n|^{5} \mid Q_{n+2}^{\lambda}-2 Q_{n+1}^{\lambda} & +Q_{n}^{\lambda} \mid \\
=\left|n^{3} Q_{n+2}^{\lambda}\right|\left|n^{3} Q_{n+1}^{\lambda}\right| & \left|n^{3} Q_{n}^{\lambda}\right| \\
\times & \left|\frac{\lambda\left(q_{n+2}-2 q_{n+1}+q_{n}\right)}{n^{4}}+\frac{q_{n}\left(q_{n+1}-q_{n+2}\right)+q_{n+2}\left(q_{n+1}-q_{n}\right)}{n^{4}}\right|
\end{aligned}
$$

we deduce from (3.31) and (3.34) that

$$
\sup _{n \in \mathbb{Z} \backslash\{0\}}|n|^{5}\left|Q_{n+2}^{\lambda}-2 Q_{n+1}^{\lambda}+Q_{n}^{\lambda}\right|<\infty .
$$

Consequently, $(\lambda-\partial F(0))^{-1} \in \mathcal{L}\left(C^{1+\delta}\left(\mathbb{S}^{1}\right), C^{4+\delta}\left(\mathbb{S}^{1}\right)\right)$ for Re $\lambda \geqslant \lambda_{*}$ by Lemma 3.7 and (3.31), (3.33), and (3.36). Since (3.31) also ensures

$$
(\lambda-\partial F(0))^{-1} \in \mathcal{L}\left(H^{s}\left(\mathbb{S}^{1}\right)\right), \quad s>0,
$$

we conclude $(\lambda-\partial F(0))^{-1} \in \mathcal{L}\left(h^{1+\delta}\left(\mathbb{S}^{1}\right), h^{4+\delta}\left(\mathbb{S}^{1}\right)\right)$ for $\operatorname{Re} \lambda \geqslant \lambda_{*}$ as in the proof of Lemma 3.8 .

The second step consists of proving the resolvent estimate

$$
|\lambda|\left\|(\lambda-\partial F(0))^{-1}\right\|_{\mathcal{L}\left(h^{1+\delta}\left(\mathbb{S}^{1}\right)\right)} \leqslant c, \quad \operatorname{Re} \lambda \geqslant \lambda_{*} .
$$

Since $\operatorname{Re} q_{n}<0$ for $|n|$ sufficiently large, elementary calculations show that

$$
\left|\lambda-q_{n}\right|^{2} \geqslant c_{0}|\lambda|^{2}, \quad \operatorname{Re} \lambda \geqslant \lambda_{*}, \quad n \in \mathbb{Z},
$$

for some $c_{0}>0$. Thus, setting $S_{n}^{\lambda}:=\lambda\left(\lambda-q_{n}\right)^{-1}$ it follows

$$
\sup _{n \in \mathbb{Z} \backslash\{0\}, \operatorname{Re} \lambda \geqslant \lambda_{*}}\left|S_{n}^{\lambda}\right|<\infty .
$$

Similarly, there is $c_{1}>0$ such that

$$
\left|\lambda-q_{n}\right|^{2} \geqslant c_{1}\left|q_{n}\right|^{2}, \quad \operatorname{Re} \lambda \geqslant \lambda_{*}, \quad n \in \mathbb{Z},
$$

and we thus obtain from

$$
|n|\left|S_{n+1}^{\lambda}-S_{n}^{\lambda}\right|=\left|S_{n+1}^{\lambda}\right|\left|\frac{n^{3}}{\lambda-q_{n}}\right|\left|\frac{q_{n+1}-q_{n}}{n^{2}}\right|
$$

together with (3.34), (3.40), and (3.41) combined with (3.31) that

$$
\sup _{n \in \mathbb{Z} \backslash\{0\}, \operatorname{Re} \lambda \geqslant \lambda_{*}}|n|\left|S_{n+1}^{\lambda}-S_{n}^{\lambda}\right|<\infty .
$$


Finally, noticing that the right hand side of

$$
\begin{aligned}
& \left|\frac{q_{n+2}-2 q_{n+1}+q_{n}}{n}\right| \\
& \leqslant\left|z_{n+2}\right|\left|\frac{\mu(n+2)-2 \mu(n+1)+\mu(n)}{n}\right|+2\left|\frac{\mu(n+1)}{n^{3}}\right| \\
& +\left|\frac{\mu(n)}{n^{3}}\right|\left|z_{n+2}-z_{n+1}\right| n^{2}
\end{aligned}
$$

is bounded by (3.32) and writing

$$
\begin{aligned}
& n^{2}\left|S_{n+2}^{\lambda}-2 S_{n+1}^{\lambda}+S_{n+2}^{\lambda}\right| \\
& =\left|S_{n+2}^{\lambda}\right|\left|n^{3} Q_{n+1}^{\lambda}\right|\left|\frac{n^{3}}{\lambda-q_{n}}\right| \frac{1}{n^{4}} \\
& \quad \times\left|\lambda\left(q_{n+2}-2 q_{n+1}+q_{n}\right)+q_{n}\left(q_{n+1}-q_{n+2}\right)+q_{n+2}\left(q_{n+1}-q_{n}\right)\right|
\end{aligned}
$$

we deduce from (3.31), (3.35), (3.39), and (3.40)

$$
\sup _{n \in \mathbb{Z} \backslash\{0\}, \operatorname{Re} \lambda \geqslant \lambda_{*}} n^{2}\left|S_{n+2}^{\lambda}-2 S_{n+1}^{\lambda}+S_{n}^{\lambda}\right|<\infty .
$$

Therefore, Lemma 3.7 and (3.40), (3.42), and (3.43) imply

$$
|\lambda|\left\|(\lambda-\partial F(0))^{-1}\right\|_{\mathcal{L}\left(C^{1+\delta}\left(\mathbb{S}^{1}\right)\right.} \leqslant c, \quad \operatorname{Re} \lambda \geqslant \lambda_{*},
$$

whence (3.38) due to (3.37). This proves the assertion.

Now we are in a position to establish a well-posedness result regarding equation (3.28).

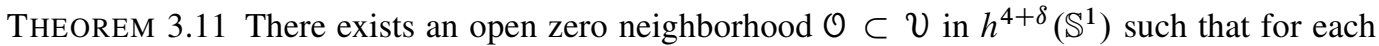
$\rho_{0} \in \mathcal{O}$ there is $T:=T\left(\rho_{0}\right)>0$ and a unique solution

$$
\rho \in C\left([0, T], \text { V) } \cap C^{1}\left([0, T], h^{1+\delta}\left(\mathbb{S}^{1}\right)\right)\right.
$$

to

$$
\partial_{t} \rho=F(\rho), \quad t>0, \quad \rho(0)=\rho_{0} .
$$

Moreover, $\rho([0, T])) \subset \mathcal{O}$.

Proof. We shall invoke [13, Thm.8.1]. Fix $\xi \in(0, \delta)$ and put $\vartheta:=(\delta-\xi) / 3$. Set

$$
E:=h^{1+\xi}\left(\mathbb{S}^{1}\right), \quad E_{0}:=h^{1+\delta}\left(\mathbb{S}^{1}\right), \quad E_{1}:=h^{4+\delta}\left(\mathbb{S}^{1}\right)
$$

in [13, Thm.8.1]. As $\delta \in(0,1)$ was arbitrary in Proposition 3.10, it follows that

$$
-\partial F(0) \in \mathcal{H}\left(h^{4+\xi}\left(\mathbb{S}^{1}\right), h^{1+\xi}\left(\mathbb{S}^{1}\right)\right) .
$$

Thus, since $\mathcal{H}\left(h^{4+\xi}\left(\mathbb{S}^{1}\right), h^{1+\xi}\left(\mathbb{S}^{1}\right)\right)$ is open in $\mathcal{L}\left(h^{4+\xi}\left(\mathbb{S}^{1}\right), h^{1+\xi}\left(\mathbb{S}^{1}\right)\right)$, there is an open zero neighborhood $U_{\xi}$ in $h^{4+\xi}\left(\mathbb{S}^{1}\right)$ such that $-\partial F(\rho) \in \mathcal{H}\left(h^{4+\xi}\left(\mathbb{S}^{1}\right), h^{1+\xi}\left(\mathbb{S}^{1}\right)\right)$ for each $\rho \in U_{\xi}$. Then 
$\mathcal{O}:=U_{\xi} \cap W$ with $W$ from Lemma 3.8 is an open zero neighborhood in $h^{4+\delta}\left(\mathbb{S}^{1}\right)$. Furthermore, $\partial F(\rho): h^{4+\delta}\left(\mathbb{S}^{1}\right) \rightarrow h^{1+\delta}\left(\mathbb{S}^{1}\right)$ for $\rho \in \mathcal{O}$ is the part of $-\partial F(\rho) \in \mathcal{H}\left(h^{4+\xi}\left(\mathbb{S}^{1}\right), h^{1+\xi}\left(\mathbb{S}^{1}\right)\right)$ in

$$
h^{1+\delta}\left(\mathbb{S}^{1}\right) \doteq\left(h^{1+\xi}\left(\mathbb{S}^{1}\right), h^{4+\xi}\left(\mathbb{S}^{1}\right)\right)_{\vartheta, \infty}^{0}
$$

with continuous interpolation functor $(\cdot, \cdot)_{\vartheta, \infty}^{0}$ and

$$
\left\{h \in h^{4+\xi}\left(\mathbb{S}^{1}\right) ; \partial F(\rho)[h] \in h^{1+\delta}\left(\mathbb{S}^{1}\right)\right\}=h^{4+\delta}\left(\mathbb{S}^{1}\right) .
$$

Now the assertion is a consequence of [13, Thm.8.1].

To finish off the proof of Theorem 2.1 let

$$
\rho \in C\left([0, T], \text { V) } \cap C^{1}\left([0, T], h^{1+8}\left(\mathbb{S}^{1}\right)\right)\right.
$$

be the solution to (3.28) for a given initial value $\rho_{0} \in \mathcal{O}$. Then

$$
Q_{\mathrm{i}}:=S\left(\rho, \partial_{t} \rho\right) \in C\left([0, T], h^{2+\delta}\left(B^{\mathrm{i}}\right)\right), \quad Q_{\mathrm{o}}:=T\left(\rho, Q_{\mathrm{i}}-\mathcal{K}(\rho)\right) \in C\left([0, T], h^{2+\delta}\left(B^{\mathrm{o}}\right)\right)
$$

by Lemma 3.3, Proposition 3.4, and Proposition 3.6. Since $\rho$ solves (3.12), it follows, for $\rho=\rho(t)$ with $t \in[0, T]$ fixed, that

$$
\frac{\phi_{*}^{\rho} \partial_{t} \rho}{\left|\nabla N_{\rho}\right|}=\frac{-1}{\left|\Theta_{\mathrm{o}}\right|^{2}}\left(\alpha_{\mathrm{o}} \partial_{v_{\rho}}\left(\phi_{*}^{\rho} Q_{\mathrm{o}}\right)+\beta_{\mathrm{o}} \partial_{\tau_{\rho}}\left(\phi_{*}^{\rho} Q_{\mathrm{o}}\right)\right) \quad \text { on } \quad \Gamma_{\rho} .
$$

Recalling from (3.9) that $\Delta\left(\phi_{*}^{\rho} Q_{\mathrm{o}}\right)=0$ and $\operatorname{div}\left(\vec{z} \times \nabla\left(\phi_{*}^{\rho} Q_{\mathrm{o}}\right)\right)=0$ in $\Omega_{\rho}^{\mathrm{o}}$, we deduce from Gauss' Theorem

$$
\frac{-1}{\left|\Theta_{\mathrm{o}}\right|^{2}} \int_{\Gamma_{\rho}}\left(\alpha_{\mathrm{o}} \partial_{\nu_{\rho}}\left(\phi_{*}^{\rho} Q_{\mathrm{o}}\right)+\beta_{\mathrm{o}} \partial_{\tau_{\rho}}\left(\phi_{*}^{\rho} Q_{\mathrm{o}}\right)\right) \mathrm{d} \sigma=\frac{-1}{\left|\Theta_{\mathrm{o}}\right|^{2}} \int_{R \mathbb{S}^{1}}\left(\alpha_{\mathrm{o}} \partial_{\nu}\left(\phi_{*}^{\rho} Q_{\mathrm{o}}\right)+\beta_{\mathrm{o}} \partial_{\tau}\left(\phi_{*}^{\rho} Q_{\mathrm{o}}\right)\right) \mathrm{d} \sigma
$$

and thus, due to $\phi_{*}^{\rho} Q_{\mathrm{o}}=Q_{\mathrm{o}}$ and (3.11) on $R \mathbb{S}^{1}$, that

$$
\int_{\Gamma_{\rho}} \frac{\phi_{*}^{\rho} \partial_{t} \rho}{\left|\nabla N_{\rho}\right|} \mathrm{d} \sigma=0, \quad t \in[0, T] .
$$

Consequently, with

$$
P_{\mathrm{i}}(t):=\phi_{*}^{\rho(t)} Q_{\mathrm{i}}(t) \in h^{2+\delta}\left(\Omega_{\rho(t)}^{\mathrm{i}}\right), \quad P_{\mathrm{o}}(t):=\phi_{*}^{\rho(t)} Q_{\mathrm{o}}(t) h^{2+\delta}\left(\Omega_{\rho(t)}^{\mathrm{o}}\right)
$$

for $t \in[0, T]$ we obtain a solution $\left(\rho, P_{\mathrm{i}}, P_{\mathrm{o}}\right)$ to (2.11)-(2.14) which is unique up to a common additive constant in the pressures $P_{\mathrm{i}}$ and $P_{\mathrm{o}}$. This yields Theorem 2.1.

\section{Proof of Theorem 2.2}

We first prove instability of the trivial solution if $\varrho_{\mathrm{i}}>\varrho_{\mathrm{o}}$ as claimed in Theorem 2.2. Recall that $\gamma_{j}:=\varrho_{j} \omega^{2} / 2$. 
THEOREM 4.1 If $\varrho_{\mathrm{i}}>\varrho_{\mathrm{o}}$, then

$$
\partial_{t} \rho=F(\rho), \quad t>0, \quad \rho(0)=\rho_{0},
$$

has backward solutions which do exponentially decay to zero. In particular, the trivial solution $\rho=0$ of this flow is unstable.

Proof. The compact embedding $h^{4+\delta}\left(\mathbb{S}^{1}\right) \hookrightarrow h^{1+\delta}\left(\mathbb{S}^{1}\right)$ and Proposition 3.10 imply that $\partial F(0)$ has compact resolvent. So the spectrum of $\partial F(0)$ consists of eigenvalues only, which, according to Proposition 3.9, are given by $\left\{q_{n} ; n \in \mathbb{Z} \backslash\{0\}\right\}$ and $q_{0}:=0$. Since $\varrho_{\mathrm{i}}>\varrho_{\mathrm{o}}$, (3.29) implies that $\mu(1)>0$, hence (3.30) shows that $\operatorname{Re} q_{1}>0$. Clearly,

$$
\inf \left\{\operatorname{Re} q_{n} ; \operatorname{Re} q_{n}>0\right\}>0 .
$$

The assertion now follows from [13, Thm.9.1.3].

To prove stability of the trivial solution if $\varrho_{\mathrm{o}}>\varrho_{\mathrm{i}}$, we need an auxiliary result.

Lemma 4.2 Let $w$ be given as in Lemma 3.8. Then

$$
\int_{\mathbb{S}^{1}}(1+\rho) F(\rho) \mathrm{d} \sigma=0, \quad \rho \in W .
$$

Proof. Fix $\rho \in W$ and set

$$
h_{0, \rho}^{1+\delta}\left(\mathbb{S}^{1}\right):=\left\{f \in h^{1+\delta}\left(\mathbb{S}^{1}\right) ; \int_{\mathbb{S}^{1}}(1+\rho) f \mathrm{~d} \sigma=0\right\} .
$$

We claim that $1-\mathbb{R}(\rho)$ is an isomorphism on $h_{0, \rho}^{1+\delta}\left(\mathbb{S}^{1}\right)$. To see this, set $T_{f}:=T(\rho, S(\rho, f))$ for $f \in h^{1+\delta}\left(\mathbb{S}^{1}\right)$. Then, as in (3.44),

$$
\int_{\mathbb{S}^{1}}(1+\rho) \mathbb{R}(\rho) f \mathrm{~d} \sigma=\int_{\mathbb{S}^{1}}(1+\rho) \mathbb{B}_{\mathrm{o}}(\rho) T_{f} \mathrm{~d} \sigma=0
$$

and Lemma 3.8 implies that $1-\mathbb{R}(\rho)$ is indeed an isomorphism on $h_{0, \rho}^{1+\delta}\left(\mathbb{S}^{1}\right)$. But, as above,

$$
\mathbb{B}_{\mathrm{o}}(\rho) T(\rho,-\mathcal{K}(\rho)) \in h_{0, \rho}^{1+\delta}\left(\mathbb{S}^{1}\right)
$$

Therefore,

$$
F(\rho)=(1-\mathbb{R}(\rho))^{-1} \mathbb{B}_{0}(\rho) T(\rho,-\mathcal{K}(\rho)) \in h_{0, \rho}^{1+\delta}\left(\mathbb{S}^{1}\right) .
$$

We conclude the proof of Theorem 2.2 by stating the stability result for which we need to define

$$
h_{0}^{s}\left(\mathbb{S}^{1}\right):=\left\{f \in h^{s}\left(\mathbb{S}^{1}\right) ; \int_{\mathbb{S}^{1}} f \mathrm{~d} \sigma=0\right\}
$$

for $s>0$. 
THEOREM 4.3 If $\varrho_{\mathrm{o}}>\varrho_{\mathrm{i}}$, the trivial solution $\rho=0$ of

$$
\partial_{t} \rho=F(\rho), \quad t>0,
$$

is stable. More precisely, there are numbers $\omega, r, M>0$ such that for each initial datum $\rho_{0} \in h_{0}^{1+\delta}\left(\mathbb{S}^{1}\right)$ with $\left\|\rho_{0}\right\|_{h^{1+\delta}\left(\mathbb{S}^{1}\right)} \leqslant r$ there is a unique global solution

$$
\rho \in C\left(\mathbb{R}^{+}, h_{0}^{4+\delta}\left(\mathbb{S}^{1}\right)\right) \cap C^{1}\left(\mathbb{R}^{+}, h_{0}^{1+\delta}\left(\mathbb{S}^{1}\right)\right)
$$

with $\rho(0)=\rho_{0}$ and

$$
\begin{aligned}
& \left\|\rho(t)\left(1+\frac{\rho(t)}{2}\right)\right\|_{h^{4+\delta}\left(\mathbb{S}^{1}\right)}+\|\dot{\rho}(t)(1+\rho(t))\|_{h^{1+\delta}\left(\mathbb{S}^{1}\right)} \\
& \quad \leqslant M e^{-\omega t}\left\|\rho_{0}\left(1+\frac{\rho_{0}}{2}\right)\right\|_{h^{4+\delta}\left(\mathbb{S}^{1}\right)}, \quad t \geqslant 0 .
\end{aligned}
$$

Proof. Letting $\zeta:=\rho+\rho^{2} / 2$ for $\rho \in W$, problem $\partial_{t} \rho=F(\rho), t>0$, is equivalent to

$$
\partial_{t} \zeta=G(\zeta), \quad t>0
$$

where $G \in C^{2}\left(Z, h^{1+\delta}\left(\mathbb{S}^{1}\right)\right)$ with $Z:=\left\{\zeta=\rho+\rho^{2} / 2 ; \rho \in W\right\}$ is given by

$$
G(\zeta):=\sqrt{1+2 \zeta} F(\sqrt{1+2 \zeta}-1) .
$$

Moreover, Lemma 4.2 implies $G \in C^{2}\left(Z_{0}, h_{0}^{1+\delta}\left(\mathbb{S}^{1}\right)\right)$ for $Z_{0}:=Z \cap h_{0}^{4+\delta}\left(\mathbb{S}^{1}\right)$. Also,

$$
\partial G(0)=\partial F(0) \in \mathcal{L}\left(h_{0}^{4+\delta}\left(\mathbb{S}^{1}\right), h_{0}^{1+\delta}\left(\mathbb{S}^{1}\right)\right) .
$$

Thus $\partial G(0)$ has compact resolvent and its (point) spectrum equals $\left\{q_{n} ; n \in \mathbb{Z} \backslash\{0\}\right\}$. Now, by (3.29),

$$
\operatorname{Re} q_{n}=\frac{A_{n}}{A_{n}^{2}+B^{2}} \mu(n), \quad n \in \mathbb{Z} \backslash\{0\},
$$

while $\varrho_{\mathrm{o}}>\varrho_{\mathrm{i}}$ implies that

$$
\mu(n)=|n|\left(\sigma-2\left(\gamma_{\mathrm{o}}-\gamma_{\mathrm{i}}\right)-\sigma n^{2}\right) \leqslant-2\left(\gamma_{\mathrm{o}}-\gamma_{\mathrm{i}}\right)<0, \quad n \in \mathbb{Z} \backslash\{0\} .
$$

This, combined with (3.30), shows that the spectrum of $\partial G(0)$ is contained in a half plane $[\operatorname{Re} \lambda \leqslant-\omega]$ for some $\omega>0$. The assertion now follows from [13, Thm.9.1.2] applied to (4.1).

Note that our analysis yields an explicit estimate of the exponential decay rate $\omega$ in terms of the physical parameters through (3.29).

\section{Equilibrium solutions}

To prove Proposition 2.3 let $\left(\Gamma, P_{\mathrm{i}}, P_{\mathrm{o}}\right)$ be any equilibrium for (2.6)-(2.9) with $\Gamma \in C^{2+\delta}$ and $P_{j} \in h^{2+\delta}\left(\Omega^{j}\right)$ for $j=\mathrm{i}, \mathrm{o}$, that is,

$$
\begin{aligned}
& \Delta P_{j}=0 \quad \text { in } \Omega^{j}, \quad j=\mathrm{i}, \mathrm{o}, \\
& P_{\mathrm{i}}-P_{\mathrm{o}}=\sigma \kappa_{\Gamma}+\left(\gamma_{\mathrm{o}}-\gamma_{\mathrm{i}}\right)|x|^{2} \quad \text { on } \Gamma \text {, } \\
& \alpha_{j} \partial_{v_{\Gamma}} P_{j}+\beta_{j} \partial_{\tau_{\Gamma}} P_{j}=0 \\
& \text { on } \Gamma, \quad j=\mathrm{i}, \mathrm{o}, \\
& \alpha_{\mathrm{o}} \partial_{\nu} P_{\mathrm{o}}+\beta_{\mathrm{o}} \partial_{\tau} P_{\mathrm{o}}=0 \\
& \text { on }[|x|=R] \text {. }
\end{aligned}
$$


Then

$$
\begin{aligned}
\Delta P_{\mathrm{i}}=0 & \text { in } \Omega^{\mathrm{i}}, \\
\alpha_{\mathrm{i}} \partial_{\nu_{\Gamma}} P_{\mathrm{i}}+\beta_{\mathrm{i}} \partial_{\tau_{\Gamma}} P_{\mathrm{i}}=0 & \text { on } \Gamma,
\end{aligned}
$$

so that Lemma 3.5 implies that $P_{\mathrm{i}} \equiv c_{\mathrm{i}}$ for some constant $c_{\mathrm{i}}$. Moreover, since also

$$
\begin{aligned}
\Delta P_{\mathrm{o}}=0 & \text { in } \Omega^{\mathrm{o}}, \\
\alpha_{\mathrm{o}} \partial_{\nu_{\Gamma}} P_{\mathrm{o}}+\beta_{\mathrm{o}} \partial_{\tau_{\Gamma}} P_{\mathrm{o}}=0 & \text { on } \Gamma, \\
\alpha_{\mathrm{o}} \partial_{\nu} P_{\mathrm{o}}+\beta_{\mathrm{o}} \partial_{\tau} P_{\mathrm{o}}=0 & \text { on } R \mathbb{S}^{1},
\end{aligned}
$$

a similar Fredholm argument as in Lemma 3.5 shows that $P_{\mathrm{o}} \equiv c_{\mathrm{o}}$ for some constant $c_{\mathrm{o}}$. If $\Gamma=\Gamma_{\rho}$ for some $\rho \in V$, then we derive equation (2.15) with $c=c_{\mathrm{i}}-c_{\mathrm{o}}$ from (5.2) and Lemma 3.3. A bootstrapping argument now shows that $\rho$ is smooth. This proves Proposition 2.3.

\section{REFERENCES}

1. Alvarez-Lacalle, E., Gadêlha, H., and Miranda, J. A., Coriolis effects on fingering patterns under rotation, Physical Review E 78 026305/1-10. MR2787492

2. Amann, H., Dual semigroups and second order linear elliptic boundary value problems. Israel J. Math. 45 (1983), 225-254. Zbl0535.35017 MR0719122

3. ARENDT, W. AND BU, S., Operator-valued Fourier multipliers on periodic Besov spaces and applications. Proc. Edinb. Math. Soc. (2) 47 (2004), 15-33. Zb11083. 42009 MR2064734

4. Chou, H.-C., Chou, F.-C., Wang, M.-W. And Tsai, C.-S., Effect of Coriolis Force on Fingering Instability and Liquid Usage Reduction, Jpn. J. Appl. Phys. 44 (2005), L606-L609.

5. Constantin, P. And Pugh, M., Global solutions for small data to the Hele-Shaw problem, Nonlinearity 6 (1987) 393-415. Zbl0808.35104 MR1223740

6. Duchon, J. AND Robert, R., Evolution d'une interface par capillarité et diffusion de volume, Ann. l'Inst. H. Poicaré 1 (1984) 361-378. Zbl0572. 35051 MR0779874

7. Ehrnström, M., EsCher, J. AND MATIOC, B.-V., Well-posedness, instabilities, and bifurcation results for the flow in a rotating Hele-Shaw cell, J. Math. Fluid Mech. 13 (2011) 271-293. MR2805866

8. Escher, J. And Simonett, G., Classical solutions for Hele-Shaw models with surface tension, Adv in Diff. Eq. 2 (1997) 619-642. Zb11023. 35527 MR1441859

9. Friedman, A. AND TAO, Y., Nonlinear stability of the Muskat problem with capillary pressure at the free boundary, Nonlinear Analysis 53 (2003) 45-80. Zbl1028.35123 MR1992404

10. Gadêlha, H., Brito, N. AND Miranda, J. A., Dynamics of viscous fingers in rotating Hele-Shaw cells with coriolis effects, Physical Review E 75 (2007) 016305/1-9.

11. Gilbarg, D. AND TRUdinger, N. S., Elliptic partial differential equations of second order, Grundlehren der Mathematischen Wissenschaften, Vol. 224, Springer-Verlag Berlin 1977. Zb10562. 35001 MR0737190

12. Hele-Shaw, H. S., The flow of water, Nature 58 (1897), 467-468, JFM 29.0646.01

13. LunARDI, A., Analytic Semigroups and Optimal Regularity in Parabolic Problems. Progress in Nonlinear Differential Equations and their Applications 16. Birkhäuser Verlag, Basel, 1995. Zb10816.35001 MR1329547

14. Saffman, P. G. AND TAYLOR, G. I., The penetration of a fluid into a porous medium or Hele-Shaw cell containing a more viscous fluid, Proc. R. Soc. A 245 (1958) 312-329. Zbl0086. 41603 MR0097227

15. Schwartz, L. W., Instability and fingering in a rotating Hele-Shaw cell or porous medium, Physics of Fluids A 1 (1989) 167-69. 
16. SChWARTZ, L. W. AND Roy, R. V., Theoretical and numerical results for spin coating of viscous liquids, Physics of Fluids 16 (2004) 569-585. Zbl1186 . 76466 MR2035460

17. Waters, S. L. And Cummings, L. J., Coriolis effects in a rotating Hele-Shaw cell, Physics of Fluids 17 (2005) 048101-048104. Zbl1187.76553 MR2136595

18. Ye, J. AND TANVEer, S., Global solutions for two-phase Hele-Shaw bubble for a near-circular initial shape, Complex Variables and Elliptic Equations 57 (2012), 23-61. Zbl1236. 35211 MR2864701 